\title{
An Efficient Algorithm for Computing High-Quality Paths amid Polygonal Obstacles
}

\author{
PANKAJ K. AGARWAL, Duke University, USA \\ KYLE FOX, The University of Texas at Dallas, USA \\ OREN SALZMAN, Carnegie Mellon University, USA
}

\begin{abstract}
We study a path-planning problem amid a set $\mathcal{O}$ of obstacles in $\mathbb{R}^{2}$, in which we wish to compute a short path between two points while also maintaining a high clearance from $\mathcal{O}$; the clearance of a point is its distance from a nearest obstacle in $\mathcal{O}$. Specifically, the problem asks for a path minimizing the reciprocal of the clearance integrated over the length of the path. We present the first polynomial-time approximation scheme for this problem. Let $n$ be the total number of obstacle vertices and let $\varepsilon \in(0,1]$. Our algorithm computes in time $O\left(\frac{n^{2}}{\varepsilon^{2}} \log \frac{n}{\varepsilon}\right)$ a path of total cost at most $(1+\varepsilon)$ times the cost of the optimal path.
\end{abstract}

CCS Concepts: • Theory of computation $\rightarrow$ Approximation algorithms analysis; Computational geometry; • Computing methodologies $\rightarrow$ Robotic planning;

Additional Key Words and Phrases: Motion planning, geometry, approximation, bicriteria objective

\section{ACM Reference format:}

Pankaj K. Agarwal, Kyle Fox, and Oren Salzman. 2018. An Efficient Algorithm for Computing High-Quality Paths amid Polygonal Obstacles. ACM Trans. Algorithms 14, 4, Article 46 (August 2018), 21 pages.

https://doi.org/10.1145/3230650

\section{INTRODUCTION}

Motivation. Robot motion planning deals with planning a collision-free path for a moving object in an environment cluttered with obstacles [7,11]. It has applications in diverse domains such as surgical planning and computational biology. Typically, a high-quality path is desired where quality can be measured in terms of path length, clearance (distance from nearest obstacle at any given time), or smoothness, to mention a few criteria.

A preliminary version of this article appeared in the Proceedings of the 27th Annual ACM-SIAM Symposium on Discrete Algorithms. Most of this work was done while K. Fox was a postdoctoral associate at Duke University and O. Salzman was a student at Tel Aviv University. Work by P.K. Agarwal and K. Fox was supported in part by NSF under Grants CCF-0940671, CCF-10-12254, CCF-11-61359, IIS-14-08846, and CCF-15-13816, and by Grant 2012/229 from the U.S.-Israel Binational Science Foundation. Work by O. Salzman was supported in part by the Israel Science Foundation under Grant 1102/11, by the German-Israeli Foundation under Grant 1150-82.6/2011, by the Hermann Minkowski-Minerva Center for Geometry at Tel Aviv University, by the National Science Foundation under Grant IIS-14-09003, by Toyota Motor Engineering \& Manufacturing (TEMA), and by the Office of Naval Research.

Authors' addresses: P. K. Agarwal, Computer Science, Mathematics, Duke University, Durham, NC, 27708-0129; email: pankaj@cs.duke.edu; K. Fox, Computer Science, The University of Texas at Dallas, Richardson, TX, 75080; email: kyle.fox@utdallas.edu; O. Salzman, Robotics Insitute, Carnegie Mellon University, Pittsburgh, PA, 15213; email: osalzman@ andrew.cmu.edu.

Permission to make digital or hard copies of all or part of this work for personal or classroom use is granted without fee provided that copies are not made or distributed for profit or commercial advantage and that copies bear this notice and the full citation on the first page. Copyrights for components of this work owned by others than ACM must be honored. Abstracting with credit is permitted. To copy otherwise, or republish, to post on servers or to redistribute to lists, requires prior specific permission and/or a fee. Request permissions from permissions@acm.org.

(c) 2018 ACM 1549-6325/2018/08-ART46 $\$ 15.00$

https://doi.org/10.1145/3230650 
Problem Statement. Let $\mathcal{O}$ be a set of polygonal obstacles in the plane, consisting of $n$ vertices in total. A path $\gamma$ for a point robot moving in the plane is a continuous function $\gamma:[0,1] \rightarrow \mathbb{R}^{2}$. Let $\|p q\|$ denote the Euclidean distance between two points $p, q$ and $\|p, o\|=\min _{q \in o}\|p, q\|$ denote the minimal Euclidean distance between $p$ and any point in obstacle $o$. The clearance of a point $p$, denoted by $\operatorname{clr}(p):=\min _{o \in \mathcal{O}}\|p o\|$, is the minimal Euclidean distance between $p$ and an obstacle $(\operatorname{clr}(p)=0$ if $p$ lies in an obstacle).

We use the following cost function, as defined by Wein et al. [20], that takes both the length and the clearance of points along a path $\gamma$ into account:

$$
\mu(\gamma):=\int_{\gamma} \frac{1}{\operatorname{clr}(\gamma(\tau))} \mathrm{d} \tau
$$

This cost function is useful in many situations because we wish to find a short path that does not pass too close to the obstacles due to safety requirements. As done by Wein et al. [20], one could also consider variants of this cost function where some positive power of the clearance is used in the denominator instead; however, we focus on the version give in Equation (1) as it greatly simplifies the algebra and is the main focus of their article as well. For two points $p, q \in \mathbb{R}^{2}$, let $\pi(p, q)$ be the minimum $\operatorname{cost}^{1}$ of any path between $p$ and $q$.

The (approximate) minimal-cost path problem is defined as follows: Given the set of obstacles $\mathcal{O}$ in $\mathbb{R}^{2}$, a real number $\varepsilon \in(0,1]$, a start position $s$, and a target position $t$, compute a path between $s$ and $t$ with cost at most $(1+\varepsilon) \cdot \pi(s, t)$.

Related Work. There is extensive work in robotics and computational geometry on computing shortest collision-free paths for a point moving amid a set of planar obstacles, and by now optimal $O(n \log n)$ algorithms are known; see Mitchell [15] for a survey and References [6, 13] for recent results. There is also work on computing paths with the minimum number of links [16]. A drawback of these paths is that they may touch obstacle boundaries and therefore their clearance may be zero. Conversely, if maximizing the distance from the obstacles is the optimization criteria, then the path can be computed by constructing a maximum spanning tree in the Voronoi diagram of the obstacles (see Ó'Dúnlaing and Yap [17]). Wein et al. [19] considered the problem of computing shortest paths where every point has clearance at least $\delta$ for some parameter $\delta$. However, this measure does not quantify the tradeoff between the length and the clearance, and the optimal path may be very long. Wein et al. [20] suggested the cost function defined in Equation (1) to balance between minimizing the path length and maximizing its clearance. They devise an approximation algorithm to compute near-optimal paths under this metric for a point robot moving amidst polygonal obstacles in the plane. Their approximation algorithm runs in time polynomial in $\frac{1}{\varepsilon}, n$, and $\Lambda$ where $\varepsilon$ is the maximal additive error, $n$ is the number of obstacle vertices, and $\Lambda$ is (roughly speaking) the total cost of the edges in the Voronoi diagram of the obstacles; for the exact definition of $\Lambda$, see Reference [20]. Note that the running time of their algorithm is exponential in the worst-case, because the value of $\Lambda$ may be exponential as a function of the input size. We are not aware of any polynomial-time approximation algorithm for this problem. It is not known whether the problem of computing the optimal path is NP-hard.

The problem of computing shortest paths amid polyhedral obstacles in $\mathbb{R}^{3}$ is NP-hard [3], and a few heuristics have been proposed in the context of sampling-based motion planning in high

\footnotetext{
${ }^{1}$ Wein et al. prove the optimality of a path with respect to a single obstacle as well as along a Voronoi edge. It remains an open question to prove that the minimal-cost path exists (amidst a set of obstacles), i.e., whether there is a limit of points at which an optimal path enters and leaves Voronoi edges. For simplicity, this article is written as if the minimal-cost path does exist.
} 
dimensions (a widely used approach in practice [7]) to compute a short path that has some clearance; see, e.g., Reference [18].

Several other bicriteria measures have been proposed in the context of path planning amid obstacles in $\mathbb{R}^{2}$, which combine the length of the path with curvature, the number of links in the path, the visibility of the path, and so on (see, e.g., References $[1,5,14]$ and references therein).

The cost function of Wein et al. [20] was recently used in the context of adaptive sampling for surface reconstruction [4]. (See also Reference [8], where a similar cost function was used for adaptive sampling.) We also note a recent work by Cohen et al. [9], which is in some sense dual to the problem studied here: Given a point set $P$ and a path $\gamma$, they define the cost of $\gamma$ to be the integral of clearance along the path, and the goal is to compute a minimal-cost path between two given points. They present an approximation algorithm whose running time is near-linear in the number of points.

Our Contribution. We present an algorithm that, given $\mathcal{O}, s, t$, and $\varepsilon \in(0,1]$, computes in time $O\left(\frac{n^{2}}{\varepsilon^{2}} \log \frac{n}{\varepsilon}\right)$ an $s, t$-path whose cost is at most $(1+\varepsilon) \pi(s, t)$.

As in Reference [20], our algorithm is based on sampling, i.e., it employs a weighted geometric graph $G=(V, E)$ with $V \subset \mathbb{R}^{2}$ and $s, t \in V$ and computes a minimum-cost $s, t$-path in $G$. However, we prove a number of useful properties of optimal paths that enable us to sample fewer points and construct a graph of size $O\left(\frac{n^{2}}{\varepsilon^{2}} \log \frac{n}{\varepsilon}\right)$.

We first compute the Voronoi diagram $\mathcal{V}$ of $\mathcal{O}$ and then refine each Voronoi cell into constant-size cells (here, the size of a cell is defined as the number cell edges). We refer to the latter as the refined Voronoi diagram of $\mathcal{O}$ and denote it by $\tilde{\mathcal{V}}$. We prove in Section 3 the existence of an $s, t$-path $\gamma$ whose cost is $O(\pi(s, t))$ and that has the following useful properties: (i) for every cell $T \in \tilde{\mathcal{V}}, \gamma \cap \operatorname{int}(T)$ is a connected subpath and the clearances of all points in this subpath are the same; we describe these subpaths as well-behaved; (ii) for every edge $e \in \tilde{\mathcal{V}}$, there are $O(1)$ points, called anchor points, that depend only on the two cells incident to $e$ with the property that either $\gamma$ intersects $e$ transversally (i.e., $\gamma \cap e$ is a single point) or the endpoints of $\gamma \cap e$ are anchor points. We use anchor points to propose a simple $O(n)$-approximation algorithm (Section 4.1). We then use anchor points and the existence of well-behaved paths to choose a set of $O(n)$ sample points on each edge of $\tilde{\mathcal{V}}$ and construct a planar graph $G$ with $O\left(n^{2}\right)$ vertices and edges so that the optimal $s, t$-path in $G$ has cost $O(\pi(s, t))$ (Section 4.2).

Finally, we prove additional properties of optimal paths to construct the final graph with $O\left(\frac{n^{2}}{\varepsilon^{2}} \log \frac{n}{\varepsilon}\right)$ edges (Section 4.3). Unlike Wein et al. [20], we do not connect every pair of sample points on the boundary of a cell $T$ of $\tilde{\mathcal{V}}$. Instead, we construct a small size spanner within $T$, which ensures that the number of edges in the graph is only $O\left(\frac{n^{2}}{\varepsilon^{2}} \log \frac{n}{\varepsilon}\right)$ and not $O\left(\frac{n^{3}}{\varepsilon^{2}}\right)$.

\section{PRELIMINARIES}

Recall that $\mathcal{O}$ is a set of disjoint polygonal obstacles in the plane consisting of $n$ vertices in total. We refer to the edges and vertices of $\mathcal{O}$ as its features. Given a point $p$ and a feature $o$, let $\psi_{o}(p)$ be the closest point to $p$ on $o$ so that $\|p o\|=\left\|p \psi_{o}(p)\right\|$. If a path $\gamma$ contains two points $p$ and $q$, we let $\gamma[p, q]$ denote the subpath of $\gamma$ between $p$ and $q$. Let $\mathcal{F}=\operatorname{cl}\left(\mathbb{R}^{2} \backslash \mathcal{O}\right)$ denote the free space. We assume in this article that the free space $\mathcal{F}$ is bounded. This assumption can be enforced by placing a sufficiently large bounding box around $\mathcal{O}$ and the points $s$ and $t$.

Voronoi Diagram and Its Refinement. The Voronoi cell of a polygon feature $o$, denoted by $\mathcal{V}(o)$, is the set of points in $\mathcal{F}$ for which $o$ is a closest feature of $\mathcal{O}$. The interiors of Voronoi cells of two different features are disjoint and the Voronoi diagram of features of $\mathcal{O}$, denoted by $\mathcal{V}$, is the planar subdivision of $\mathcal{F}$ induced by the Voronoi cells of features in $\mathcal{O}$. The edge between the Voronoi cells 


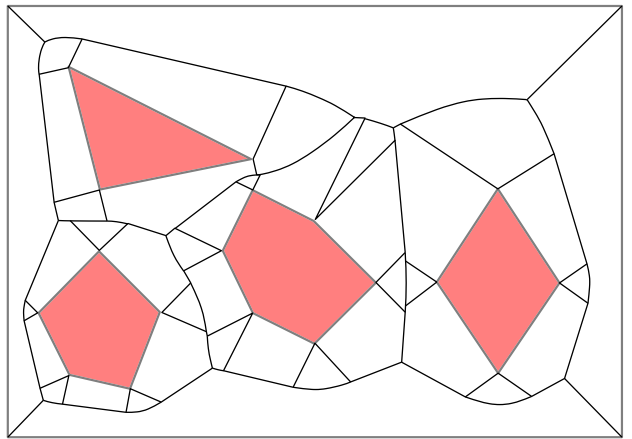

(a) Voronoi Diagram

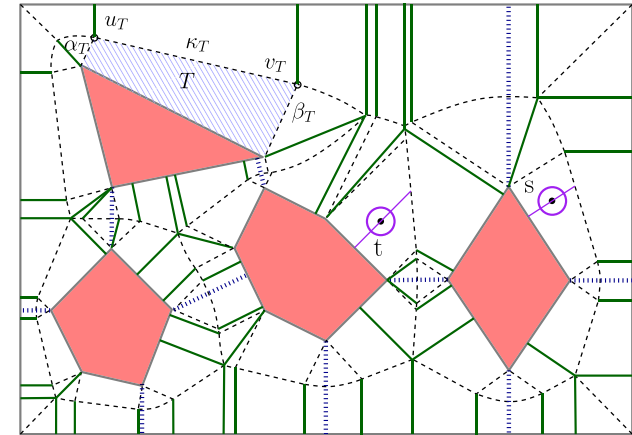

(b) Refined Voronoi Diagram

Fig. 1. The Voronoi diagram and the refined Voronoi diagram of a set of obstacles (dark red). (a) Voronoi edges are depicted by solid black lines. (b) Voronoi edges are depicted by dashed black lines. Green solid lines and blue dotted lines represent edges of type (i) and type (ii), respectively. A representative cell $T$ is depicted in light blue.

of a vertex and an edge feature is a parabolic arc, and the edge between two vertex or two edge features is a line segment. See Figure 1(a). The Voronoi diagram has total complexity $O(n)$. See Reference [2] for details.

For any obstacle feature $o$ and for any point $x$ along any edge on $\partial \mathcal{V}(o)$, the function $\left\|x \psi_{o}(x)\right\|$ is convex. We construct the refined Voronoi diagram $\tilde{\mathcal{V}}$ by adding the following edges to each Voronoi cell $\mathcal{V}(o)$ and refining it into constant-size cells:

(i) the line segments $x \psi_{o}(x)$ between each obstacle feature $o$ and every vertex $x$ on $\mathcal{V}(o)$ and

(ii) for each edge $e$ of $\mathcal{V}(o)$, the line segment $x \psi_{o}(x)$, where $x \in e$ is the point that minimizes $\left\|x \psi_{o}(x)\right\|$.

We also add a line segment from the obstacle feature $o$ closest to $s$ (resp. $t$ ) that initially follows $\psi_{o}(s) s$ (resp. $\left.\psi_{o}(t) t\right)$ and ends at the first Voronoi edge it intersects. Note that some edges of type (i) may already be present in the Voronoi diagram $\mathcal{V}$. We say that an edge in $\tilde{\mathcal{V}}$ is an internal edge if it separates two cells incident to the same polygon. Other edges are called external edges.

Clearly, the complexity of $\tilde{\mathcal{V}}$ is $O(n)$. Moreover, each cell $T$ in $\tilde{\mathcal{V}}$ is incident to a single obstacle feature $o$ and has three additional edges. One edge is external, and it is a parabolic arc or line segment that is monotone with regard to the clearance of its points. The other two edges are internal edges on $T$, and they are both line segments. For each cell $T$, let $\kappa_{T}$ denote the external edge of $T$, let $\alpha_{T}$ and $\beta_{T}$ denote the shorter and longer internal edges of $T$, respectively, and let $u_{T}$ and $v_{T}$ denote the vertices connecting $\alpha_{T}$ and $\beta_{T}$ to $\kappa_{T}$, respectively. See Figure 1(b).

For any value $c>0$, the set of points in Voronoi cell $T$ of clearance $c$, if nonempty, forms a connected arc $\eta$, which is a circular arc centered at $o$ if $o$ is a vertex and a line segment parallel to $o$ if $o$ is an edge. One endpoint of $\eta$ lies on $\beta_{T}$ and the other on $\alpha_{T}$ or $\kappa_{T}$.

Properties of Optimal Paths. We list several properties of our cost function. For detailed explanations and proofs, the reader is referred to Wein et al. [20]. Let $s=r_{s} e^{i \theta_{s}}$ be a start position and $t=r_{t} e^{i \theta_{t}}$ be a target position.

(P1) Let $o$ be a point obstacle with $\mathcal{O}=\{o\}$, and assume without loss of generality that $o$ lies at the origin and $0 \leq \theta_{s} \leq \theta_{t} \leq \pi$. The optimal path between $s$ and $t$ (see Figure 2(a)) is a 


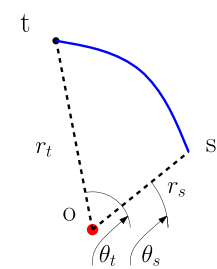

(a) Point obstacle

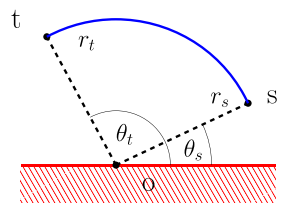

(b) Line obstacle

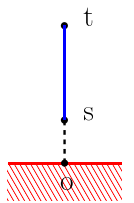

(c) Line obstacle (degenerate)

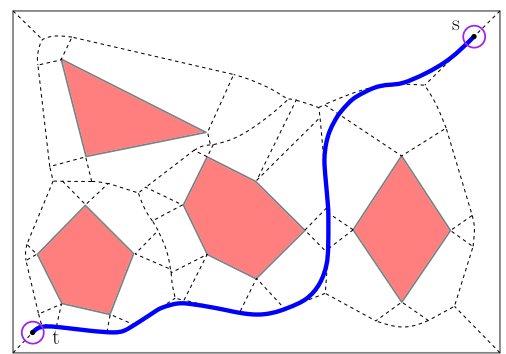

(d) Polygonal obstacles

Fig. 2. Different examples of optimal paths (blue) among different types obstacles (red). In (d), the Voronoi diagram of the obstacles is depicted by dashed black lines.

logarithmic spiral centered on $o$, and its cost is

$$
\pi(s, t)=\sqrt{\left(\theta_{t}-\theta_{s}\right)^{2}+\left(\ln r_{t}-\ln r_{s}\right)^{2}} .
$$

(P2) Let $o$ be a line obstacle with $\mathcal{O}=\{o\}$, and assume without loss of generality that $o$ is supported by the line $y=0,0 \leq \theta_{s} \leq \theta_{t} \leq \pi$, and $r_{s}=r_{t}=r$. The optimal path between $s$ and $t$ (see Figure 2(b)) is a circular arc with its center at the origin, and its $\operatorname{cost}^{2}$ is

$$
\pi(s, t)=\ln \frac{1-\cos \theta_{t}}{\sin \theta_{t}}-\ln \frac{1-\cos \theta_{s}}{\sin \theta_{s}}=\ln \tan \frac{\theta_{t}}{2}-\ln \tan \frac{\theta_{s}}{2} .
$$

(P3) Let $o$ be an obstacle with $\mathcal{O}=\{o\}$ and assume that $s$ lies on the line segment between $\psi_{o}(t)$ and $t$. The optimal path between $s$ and $t$ (see Figure 2(c)) is a line segment, and its cost is

$$
\pi(s, t)=\ln \operatorname{clr}(t)-\ln \operatorname{clr}(s) .
$$

(P4) The minimal-cost path $\gamma$ between two points $p$ and $q$ on an edge $e$ of $\mathcal{V}$ is the piece of $e$ between $p$ and $q$. Moreover, there is a closed-form formula describing the cost of $\gamma$.

(P5) Since each point within a single Voronoi cell is closest to exactly one obstacle feature, we may conclude the following: Given a set of obstacles, the optimal path connecting $s$ and $t$ consists of a sequence of circular arcs, pieces of logarithmic spirals, line segments, and pieces of Voronoi edges. Each member of this sequence begins and ends on an edge or vertex of $\tilde{\mathcal{V}}$ (see Figure 2(d)).

The following lemmas follow immediately from (P1)-(P3).

\footnotetext{
${ }^{2}$ The original equation describing the cost of the optimal path in the vicinity of a line obstacle had the obstacle on $x=0$, and it contained a minor inaccuracy in its calculation. We present the correct cost in Equation (3).
} 
Lemma 2.1. Let $p$ and $q$ be two points such that $\operatorname{clr}(p) \leq \operatorname{clr}(q)$. The following properties hold:

(i) We have $\pi(p, q) \geq \ln \frac{\operatorname{clr}(q)}{\operatorname{cr}(p)}$. If $p$ and $q$ lie in the same refined Voronoi cell of an obstacle feature o in $\tilde{\mathcal{V}}$ and if p lies on the line segment $q \psi_{o}(q)$, then the bound is tight.

(ii) If there is a single point obstacle o located at the origin, $p=r_{p} e^{i \theta_{p}}$ and $q=r_{q} e^{i \theta_{q}}$ with $0 \leq$ $\theta_{p} \leq \theta_{q} \leq \pi$, then $\pi(p, q) \geq \theta_{q}-\theta_{p}$. If $r_{p}=r_{q}$ (namely, $p$ and $q$ are equidistant to $o$ ), then the bound is tight.

Lemma 2.2. Letp be a point such that $p \in \alpha_{T}$ or $p \in \beta_{T}$ for some Voronoi cellT in $\tilde{\mathcal{V}}$. Let $w$ be another point on the same edge of T as $p$, and let $q$ be any other point on $\partial T$ such that $\operatorname{clr}(p) \leq \operatorname{clr}(w) \leq \operatorname{clr}(q)$. Then $\pi(p, w) \leq \pi(p, q)$.

Model of Computation. We are primarily concerned with the combinatorial time complexity of our algorithm. Therefore, we assume a model of computation that allows us to evaluate basic trigonometric and algebraic expressions, such as the ones given above, in constant time. Our model also allows us to find the roots of a constant-degree polynomial in constant time.

\section{WELL-BEHAVED PATHS}

Let $T$ be a cell of $\tilde{\mathcal{V}}$ incident to obstacle feature $o$, and let $p$ and $q$ be two points on $\partial T$. We define a well-behaved path between $p$ and $q$, denoted by $\gamma(p, q)$, whose cost is at most $11 \pi(p, q)$ and that can be computed in $O(1)$ time. We first define $\gamma(p, q)$, then analyze its cost, and finally prove an additional property of $\gamma(p, q)$ that allows us to compute it in $O(1)$ time.

If both $p$ and $q$ lie on the same edge of $\partial T$ or neither of them lies on the edge $\beta_{T}$, then we define $\gamma(p, q)$ to be the unique $p, q$-path along $\partial T$ that does not intersect $o$. If one of $p$ and $q$, say, $p$, lies on $\beta_{T}$, then $\gamma(p, q)$ is somewhat more involved, because the path along $\partial T$ can be quite expensive. Instead, we let $\gamma(p, q)$ enter the interior of $T$. For a point $w \in \beta_{T}$, let $\eta_{w}$ be the set of points in $T$ of the same clearance as $w$, i.e., $\eta_{w}=\{z \in T \mid \operatorname{clr}(z)=\operatorname{clr}(w)\}$.

By the discussion in Section 2, $\eta_{w}$ is a line segment or a circular arc with $w$ as one of its endpoints. Let $\bar{w}$ be the other endpoint of $\eta_{w}$. We define the path

$$
\lambda(p ; w)=p w \circ \eta_{w}
$$

to be the segment of $p w$ followed by the arc $\eta_{w}$. We refer to $w$ as the anchor point of $\lambda(p ; w)$. Let $w_{p}^{*}$ be the anchor point on edge $\beta_{T}$ of clearance greater than $\operatorname{clr}(p)$ that minimizes the cost of $\lambda(p ; w)$. Namely,

$$
w_{p}^{*}=\underset{\substack{w \in \beta_{T} \\ \operatorname{crr}(w) \geq \operatorname{clr}(p)}}{\operatorname{argmin}} \mu(\lambda(p ; w))
$$

We now define

$$
\begin{aligned}
\gamma(p, q ; w) & =\lambda(p ; w) \circ \gamma(\bar{w}, q), \\
\gamma(p, q) & =\gamma\left(p, q ; w_{p}^{*}\right) .
\end{aligned}
$$

See Figure 3.

The next two lemmas bound the cost of $\gamma(p, q)$.

LEMMA 3.1.

(i) If $p$ and $q$ lie on the same edge of $\partial T$, then $\mu(\gamma(p, q))=\pi(p, q)$.

(ii) If neither $p$ nor $q$ lies on $\beta_{T}$, then $\mu(\gamma(p, q)) \leq 3 \pi(p, q)$. 


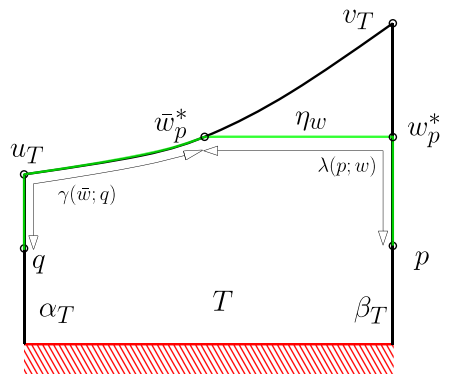

Fig. 3. Components of the well-behaved path $\gamma(p, q)$.

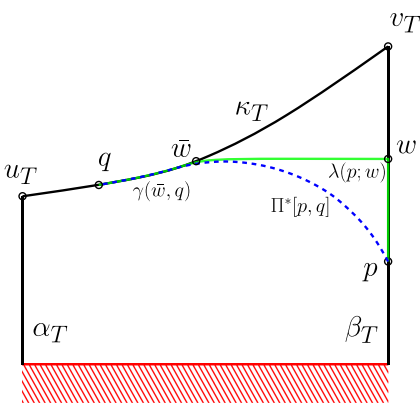

(a) Case 1: $q \in \kappa_{T}$

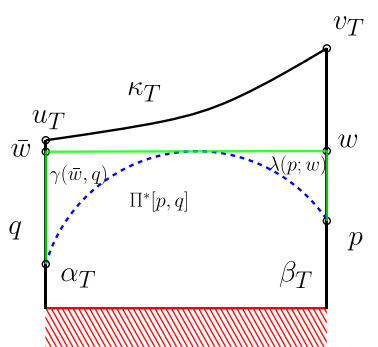

(b) Case 2: $q, \bar{w} \in \alpha_{T}$

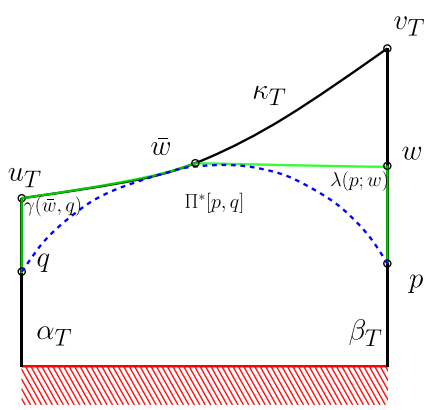

(c) Case 3: $q \in \alpha_{T}, \bar{w} \in \kappa_{T}$

Fig. 4. Different cases considered in the proof of Lemma 3.2. We use $\Pi^{*}[p, q]$ to denote the minimal-cost path between $p$ and $q$.

Proof.

(i) If $p$ and $q$ lie on the same edge $e$ of $\partial T$, then $\gamma(p, q) \subseteq e$, and by (P4), $\gamma(p, q)$ is the optimal path between $p$ and $q$. Hence, the claim follows.

(ii) Suppose $p \in \alpha_{T}$ and $q \in \kappa_{T}$. Path $\gamma(p, q)$ travels along $\alpha_{T}$ from $p$ to $u_{T}$, and then along $\kappa_{T}$ from $u_{T}$ to $q$. By Lemma 2.2 and the fact that $u_{T}$ is the lowest clearance point on $\kappa_{T}$, we have $\pi\left(p, u_{T}\right) \leq \pi(p, q)$. By the triangle inequality, we have that

$$
\pi\left(u_{T}, q\right) \leq \pi\left(u_{T}, p\right)+\pi(p, q) \leq 2 \pi(p, q) .
$$

Finally,

$$
\mu(\gamma(p, q))=\pi\left(p, u_{T}\right)+\pi\left(u_{T}, q\right) \leq 3 \pi(p, q) .
$$

Lemma 3.2. If $p \in \beta_{T}$ and $q \notin \beta_{T}$, then $\mu(\gamma(p, q)) \leq 11 \pi(p, q)$.

Proof. Let $w$ be any point of $\beta_{T}$ such that $\operatorname{clr}(w) \geq \operatorname{clr}(p)$. We begin by proving $\mu(\gamma(p, q ; w)) \leq$ $4 \mu(\lambda(p ; w))+3 \pi(p, q)$. Later, we will show $\mu\left(\lambda\left(p ; w_{p}^{*}\right)\right) \leq 2 \pi(p, q)$, proving the lemma.

To prove the first claim, we consider different cases depending on the edges of $\partial T$ that contain $\bar{w}$ and $q$. See Figure 4.

Case 1: $q \in \kappa_{T}$. In this case, $\gamma(\bar{w}, q) \subseteq \kappa_{T}$, and therefore $\mu(\gamma(\bar{w}, q))=\pi(\bar{w}, q)$. By the triangle inequality, $\pi(\bar{w}, q) \leq \mu(\lambda(p ; w))+\pi(p, q)$.

Case 2: $q, \bar{w} \in \alpha_{T}$. In this case, $\gamma(\bar{w}, q) \subseteq \alpha_{T}$, and therefore $\mu(\gamma(\bar{w}, q))=\pi(\bar{w}, q)$. Again, $\pi(\bar{w}, q) \leq$ $\mu(\lambda(p ; w))+\pi(p, q)$. 
Case 3: $q \in \alpha_{T}, \bar{w} \in \kappa_{T}$. In this case, $\gamma(\bar{w}, q)$ first travels along $\kappa_{T}$ from $\bar{w}$ to $u_{T}$ and then along $\alpha_{T}$ from $u_{T}$ to $q$. Since $\operatorname{clr}\left(u_{T}\right) \leq \operatorname{clr}(w)$,

$$
\pi\left(q, u_{T}\right) \leq \pi(q, \bar{w}) \leq \pi(q, p)+\pi(p, \bar{w}) \leq \pi(p, q)+\mu(\lambda(p ; w))
$$

by Lemma 2.2. Furthermore, by the triangle inequality,

$$
\begin{aligned}
\pi\left(\bar{w}, u_{T}\right) & \leq \mu(\lambda(p ; w))+\pi(p, q)+\pi\left(q, u_{T}\right) \\
& \leq 2 \mu(\lambda(p ; w))+2 \pi(p, q) .
\end{aligned}
$$

Hence, $\mu(\gamma(\bar{w}, q))=\pi\left(\bar{w}, u_{T}\right)+\pi\left(u_{T}, q\right) \leq 3 \mu(\lambda(p ; w))+3 \pi(p, q)$.

Since $\mu(\gamma(\bar{w}, q)) \leq 3 \mu(\lambda(p ; w))+3 \pi(p, q)$ in all three cases, $\mu(\gamma(p, q ; w)) \leq 4 \mu(\lambda(p ; w))+$ $3 \pi(p, q)$ as claimed.

Let $c^{*}$ be the maximum clearance of a point on the optimal path between $p$ and $q$ (if there are multiple optimal paths between $p$ and $q$, choose one of them arbitrarily). Let $w \in \beta_{T}$ be the point of clearance $\min \left\{c^{*}, \operatorname{clr}\left(v_{T}\right)\right\}$. We now prove that $\mu(\lambda(p ; w)) \leq 2 \pi(p, q)$.

We first note that $\operatorname{clr}(p) \leq \operatorname{clr}(w) \leq c^{*}$. Therefore, by Lemma $2.2, \mu(p w)=\pi(p, w) \leq \pi(p, q)$. Next, we argue that $\mu\left(\eta_{w}\right) \leq \pi(p, q)$. Indeed, if $o$ is a polygon edge, then $\eta_{w}$ is the Euclidean shortest path between any pair of points, one on $\beta_{T}$ and one on either $\alpha_{T}$ or $\kappa_{T}$, whose clearance never exceeds $c^{*}$. It also (trivially) has the highest clearance of any such path. If $o$ is a polygon vertex, then $\eta_{w}$ spans a shorter angle relative to $o$ than any other path whose clearance never exceeds $c^{*}$. By Lemma 2.1(ii), the cost of any such path from $\beta_{T}$ to one of $\alpha_{T}$ or $\kappa_{T}$ is at least this angle, and by (P1), the cost of $\eta_{w}$ is exactly this lower bound. Either way, any path between $p$ and $q$ also goes between $\beta_{T}$ and one of $\alpha_{T}$ or $\kappa_{T}$, so we conclude that $\mu\left(\eta_{w}\right) \leq \pi(p, q)$. Hence, $\mu(\lambda(p ; w)) \leq 2 \pi(p, q)$. In particular, $\mu\left(\lambda\left(p ; w_{p}^{*}\right)\right) \leq 2 \pi(p, q)$, and $\mu\left(\gamma\left(p, q ; w_{p}^{*}\right)\right) \leq 11 \pi(p, q)$.

If $p \in \beta_{T}$ and $q \notin \beta_{T}$, then computing $\gamma(p, q)$ requires computing the anchor point $w$ that minimizes $\mu(\lambda(p, w))$. We show that the point $w_{p}^{*} \in \beta_{T}$ that defines $\gamma(p, q)$ is either $p$ itself or a point that only depends on the geometry of $\partial T$ and not on $p$ or $q$.

Lemma 3.3. There exist two points $w_{\alpha_{T}}$ and $w_{\kappa_{T}}$ on $\beta_{T}$, such that for any $p \in \beta_{T}, w_{p}^{*} \in$ $\left\{p, w_{\alpha_{T}}, w_{\kappa_{T}}\right\}$. Furthermore, these two points can be computed in $O(1)$ time.

Proof. There are several cases to consider depending on whether $o$ is a vertex or an edge, whether the point $\bar{w}_{p}^{*}$ lies on $\kappa_{T}$ or $\alpha_{T}$, and whether $\kappa_{T}$ is a line segment or a parabolic arc. Depending on the geometry of $T$, we define $w_{\alpha_{T}}$ and $w_{\kappa_{T}}$ accordingly. In each case, we parameterize the anchor point $w$ on $\beta_{T}$ appropriately and show that $w_{p}^{*} \in\left\{p, w_{\alpha_{T}}, w_{\kappa_{T}}\right\}$. For simplicity, for a parameterized anchor point $w(t)$, we use $\eta(t), \lambda(t)$, and $\mu(t)$ to denote $\eta_{w(t)}, \lambda(p ; w(t))$, and $\mu(\lambda(p ; w(t)))$, respectively. We now describe each case:

Case 1: $o$ is a vertex. Without loss of generality, assume that $o$ lies at the origin, edges $\alpha_{T}$ and $\beta_{T}$ intersect the line $y=0$ at the origin with angles $\theta_{\alpha}$ and $\theta_{\beta}$, respectively, and $\theta_{\beta}>\theta_{\alpha} \geq 0$. In this case, $\eta_{w}$, the constant-clearance path anchored at $w \in \beta_{T}$, is a circular arc. We consider two cases depending on whether $\bar{w}_{p}^{*}$ lies on $\alpha_{T}$ or $\kappa_{T}$. See Figure 5.

Case 1(a): $\bar{w}_{p}^{*} \in \alpha_{T}$. We parameterize the anchor point $w$ by its clearance, i.e., $\operatorname{cl}(w(t))=$ $t$. Because $\operatorname{clr}\left(\bar{w}_{p}^{*}\right) \geq \operatorname{clr}(p)$ and $\bar{w}_{p}^{*} \in \alpha_{T}$, we call $t$ feasible if $t \in\left[\operatorname{clr}(p), \operatorname{clr}\left(u_{T}\right)\right]$. As $\bar{w}(t) \in \alpha_{T}$, then the cost of $\eta(t)$ is simply $\theta_{\beta}-\theta_{\alpha}$, and

$$
\mu(t)=\ln \frac{\operatorname{clr}(w(t))}{\operatorname{clr}(p)}+\theta_{\beta}-\theta_{\alpha}=\ln \frac{t}{\operatorname{clr}(p)}+\theta_{\beta}-\theta_{\alpha} .
$$

Therefore, $\mu$ is minimized in the range $\left[\operatorname{clr}(p), \operatorname{clr}\left(u_{T}\right)\right]$ for $t=\operatorname{clr}(p)$, so $w^{*}=p$ in this case. 


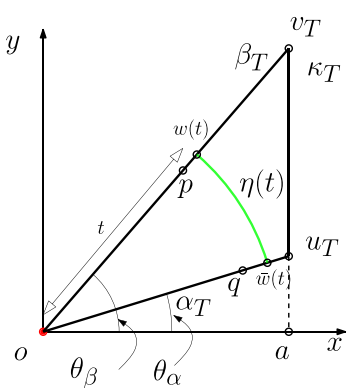

(a) Case 1(a): $\bar{w}_{p}^{*} \in \alpha_{T}$

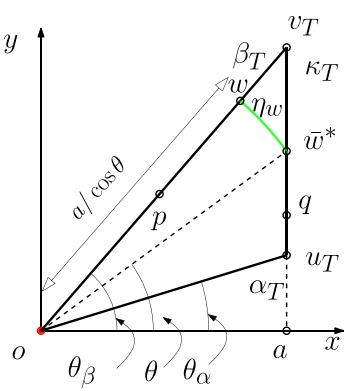

(b) Case $1(\mathrm{~b})(\mathrm{i}): \bar{w}_{p}^{*} \in \kappa_{T} ; \kappa_{T}$ is a line segment

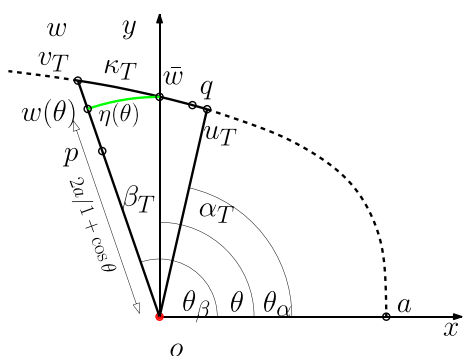

(c) Case $1(\mathrm{~b})(\mathrm{ii}): \bar{w}_{p}^{*} \in \kappa_{T} ; \kappa_{T}$ is a parabolic arc

Fig. 5. Different cases considered in the proof of Lemma 3.3, Case 1: $o$ is a vertex.

Case $1(\mathrm{~b}): \bar{w}_{p}^{*} \in \kappa_{T}$. We parameterize $w$ with the angle $\theta$ of the segment $o \bar{w}$. We call $\theta$ feasible if $\operatorname{clr}(w(\theta)) \geq \operatorname{clr}(p)$ and $\theta_{\alpha} \leq \theta \leq \theta_{\beta}$. We divide this case further into two subcases:

Case 1(b)(i): $\kappa_{T}$ is a line segment. Without loss of generality, $\kappa_{T}$ is supported by the line $x=a$. The equation of the line in polar coordinates is $r=$ $a / \cos \theta$. We have $\theta_{\beta} \leq \pi / 2$. Restricting ourselves to feasible values of $\theta$, we have

$$
\mu(\theta)=\ln \frac{\operatorname{clr}(w(\theta))}{\operatorname{cl}(p)}+\theta_{\beta}-\theta=\ln \frac{a / \cos \theta}{\operatorname{cl}(p)}+\theta_{\beta}-\theta .
$$

Taking the derivative, we obtain

$$
\frac{\mathrm{d}}{\mathrm{d} \theta} \mu(\theta)=\tan \theta-1
$$

This expression is negative for $\theta=0$, positive near $\theta=\pi / 2$, and it has at most one root within the feasible values of $\theta$, specifically, at $\theta=\pi / 4$. Therefore, $\mu(\theta)$ is minimized when either $\operatorname{clr}(w(\theta))=\operatorname{clr}(p)$ or $\theta=\theta^{*}=$ $\min \left\{\max \left\{\pi / 4, \theta_{\alpha}\right\}, \theta_{\beta}\right\}$. We pick $w_{\kappa_{T}}=w\left(\theta^{*}\right)$.

Case 1(b)(ii): $\kappa_{T}$ is a parabolic arc. Without loss of generality, the parabola supporting $\kappa_{T}$ is equidistant between $o$ and the line $x=2 a$. The equation of the parabola in polar coordinates is $r=2 a /(1+\cos \theta)$. We have $\theta \leq \pi$. The polar coordiantes of $w(\theta)$ are $w(\theta)=\left(\frac{2 a}{1+\cos \theta}, \theta_{\beta}\right)$.

Restricting ourselves to feasible values of $\theta$, we have

$$
\mu(\theta)=\ln \frac{\operatorname{clr}(w(\theta))}{\operatorname{cl}(p)}+\theta_{\beta}-\theta=\ln \frac{2 a /(1+\cos \theta)}{\operatorname{clr}(p)}+\theta_{\beta}-\theta .
$$

Here,

$$
\frac{\mathrm{d}}{\mathrm{d} \theta} \mu(\theta)=\frac{\sin \theta}{1+\cos \theta}-1=\tan (\theta / 2)-1
$$

Again, the expression is negative for $\theta=0$, positive for $\theta$ near $\pi$, and it has at most one root within the feasible values of $\theta$, specifically, at $\theta=$ $\pi / 2$. Therefore, $\mu(\theta)$ is minimized when either $\operatorname{clr}(w(\theta))=\operatorname{clr}(p)$ or $\theta=$ $\theta^{*}=\min \left\{\max \left\{\pi / 2, \theta_{\alpha}\right\}, \theta_{\beta}\right\}$. We pick $w_{\kappa_{T}}=w\left(\theta^{*}\right)$. 


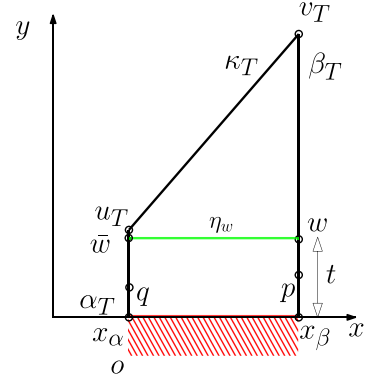

(a) Case 2(a): $\bar{w}_{p}^{*} \in \alpha_{T}$

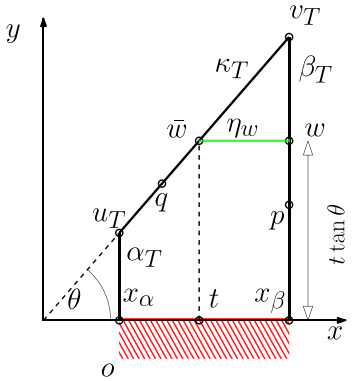

(b) Case 2(b)(i): $\bar{w}_{p}^{*} \in \kappa_{T} ; \kappa_{T}$ is a line segment

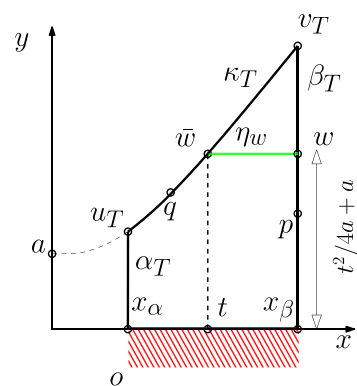

(c) Case 2(b)(ii): $\bar{w}_{p}^{*} \in \kappa_{T} ; \kappa_{T}$ is a parabolic arc

Fig. 6. Different cases considered in the proof of Lemma 3.3, Case 2: $o$ is an edge.

Case 2: $o$ is an edge. Without loss of generality, $o$ lies on the line $y=0$, the edge $\alpha_{T}$ lies on the line $x=x_{\alpha}$, the edge $\beta_{T}$ lies on the line $x=x_{\beta}$, and $x_{\beta}>x_{\alpha} \geq 0$. In this case, $\eta_{w}$ is a horizontal segment. We again consider two cases. See Figure 6.

Case 2(a): $\bar{w}_{p}^{*} \in \alpha_{T}$. As in Case 1(a), we parameterize the anchor point $w$ by its clearance, i.e., $\operatorname{clr}(w(t))=t$ where $t$ is feasible if $t \in\left[\operatorname{clr}(p), \operatorname{clr}\left(u_{T}\right)\right]$. Restricting ourselves to feasible values of $w(t)$, we have

$$
\mu(\gamma(p, \lambda))=\ln \frac{\operatorname{clr}(w(t))}{\operatorname{cl}(p)}+\frac{\|\eta(t)\|}{\operatorname{clr}(w(t))}=\ln \frac{t}{\operatorname{cl}(p)}+\frac{x_{\beta}-x_{\alpha}}{t} .
$$

We have

$$
\frac{\mathrm{d}}{\mathrm{d} t} \mu(t)=1 / t-\frac{\left(x_{\beta}-x_{\alpha}\right)}{t^{2}} .
$$

This expression is negative for $t$ near 0 , positive for large $t$, and it has at most one root within the feasible values of $t$ located at $t=x_{\beta}-x_{\alpha}$. If $x_{\beta}-x_{\alpha} \leq \operatorname{clr}\left(u_{T}\right)$, then $\mu(t)$ is minimized when either $\operatorname{clr}(w(t))=\operatorname{clr}(p)$ or $t=t^{*}=x_{\beta}-x_{\alpha}$. If $x_{\beta}-$ $x_{\alpha}>\operatorname{clr}\left(u_{T}\right)$, then $\bar{w}_{p}^{*} \in \kappa_{T}$, so assume that $x_{\beta}-x_{\alpha} \leq \operatorname{clr}\left(u_{T}\right)$. We pick $w_{\alpha_{T}}=$ $w\left(t^{*}\right)$.

Case 2(b): $\bar{w}_{p}^{*} \in \kappa_{T}$. We parameterize $w$ by the $x$-coordinate of $\bar{w}$. We call $t$ feasible if $\operatorname{cl} r(w(t)) \geq \operatorname{clr}(p)$ and $t \in\left[x_{\alpha}, x_{\beta}\right]$. There are two subcases.

Case 2(b)(i): $\kappa_{T}$ is a line segment. Without loss of generality, the line supporting $\kappa_{T}$ intersects $o$ at the origin with angle $\theta$. Restricting ourselves to feasible values of $t$, we have

$$
\mu(t)=\ln \frac{\operatorname{clr}(w(t))}{\operatorname{cl}(p)}+\frac{\|\eta(t)\|}{\operatorname{cln}(w(t))}=\ln \frac{t \tan \theta}{\operatorname{cln}(p)}+\frac{x_{\beta}-t}{t \tan \theta} .
$$

We see

$$
\frac{\mathrm{d}}{\mathrm{d} t} \mu(t)=\frac{1}{t}-\frac{x_{\beta}}{t^{2} \tan \theta}=\frac{t \tan \theta-x_{\beta}}{t^{2} \tan \theta} .
$$

This expression is negative for $t$ near 0 , positive for large $t$, and it has at most one root within feasible values of $t$, namely at $t=x_{\beta} / \tan \theta$. Therefore, $\mu(t)$ is minimized when either $\operatorname{clr}(w(t))=\operatorname{clr}(p)$ or $t=t^{*}=$ $\min \left\{\max \left\{x_{\beta} / \tan \theta, x_{\alpha}\right\}, x_{\beta}\right\}$. We pick $w_{\kappa_{T}}=w\left(t^{*}\right) ;$ note that $\operatorname{clr}\left(w_{\kappa_{T}}\right)=$ $t^{*} \tan \theta$. 
Case 2(b)(ii): $\kappa_{T}$ is a parabolic arc. Without loss of generality, the parabola supporting $\kappa_{T}$ is equidistant between $o$ and a point located at $(0,2 a)$. Therefore, the parabola is described by the equation $y=x^{2} /(4 a)+a$.

Restricting ourselves to feasible values of $t$, we have

$$
\mu(t)=\ln \frac{\operatorname{clr}(w(t))}{\operatorname{cl}(p)}+\frac{\|\eta(t)\|}{\operatorname{cl}(w(t))}=\ln \frac{t^{2} /(4 a)+a}{\operatorname{cl}(p)}+\frac{x_{\beta}-t}{t^{2} /(4 a)+a} .
$$

We have

$$
\frac{\mathrm{d}}{\mathrm{d} t} \mu(t)=\frac{2 t^{3}+4 a t^{2}+8 a\left(a-x_{\beta}\right) t-16 a^{3}}{\left(t^{2}+4 a^{2}\right)^{2}} .
$$

This expression is negative for $t$ near 0 (such $t$ may not be feasible) and positive for large $t$. The derivative of the numerator is $6 t^{2}+8 a t+$ $8 a\left(a-x_{\beta}\right)$, which has at most one positive root. Therefore, the numerator has at most one positive local maximum or minimum. We see that $\frac{\mathrm{d}}{\mathrm{d} t} \mu(t)$ goes from negative to positive around exactly one positive root (which, again, may not be feasible), and $\mu(t)$ has one minimum at a positive value of $t$. Let $t^{\prime}$ be this root of $\frac{\mathrm{d}}{\mathrm{d} t} \mu(t)$. Value $\mu(t)$ is minimized when either $\operatorname{clr}(w(t))=\operatorname{clr}(p)$ or $t=t^{*}=\min \left\{\max \left\{t^{\prime}, x_{\alpha}\right\}, x_{\beta}\right\}$. We pick $w_{\kappa_{T}}=w\left(t^{*}\right)$; note that $\operatorname{clr}\left(w_{\kappa_{T}}\right)=\left(t^{*}\right)^{2} /(4 a)+a$.

We note that in all cases $w^{*} \in\left\{p, w_{\kappa_{T}}, w_{\alpha_{T}}\right\}$ for some choices of $w_{\kappa_{T}}$ and $w_{\alpha_{T}}$ that depend only on the geometry of $T$ and not on $p$. Note that no subcase of Case 1 required picking a concrete $w_{\alpha_{T}}$, so if $o$ is a vertex, we let $w_{\alpha_{T}}$ be an arbitrary point on $\beta_{T}$. In every case, $w_{\kappa_{T}}$ and $w_{\alpha_{T}}$ can be computed in $O(1)$ time. We conclude the proof of the lemma.

\section{APPROXIMATION ALGORITHMS}

In this section, we propose a near-quadratic-time $(1+\varepsilon)$-approximation algorithm for computing the minimum-cost path between two points $s, t \in \mathcal{F}$ amid $\mathcal{O}$. We assume that $\operatorname{clr}(s) \leq \operatorname{clr}(t)$ throughout this section. We first give a high-level overview of the algorithm and then describe each step in detail. Throughout this section, let $\Pi^{*}$ denote a minimum-cost $(s, t)$-path.

High-Level Description. Our algorithm begins by computing the refined Voronoi diagram $\tilde{\mathcal{V}}$ of $\mathcal{O}$. The algorithm then works in three stages. The first stage computes an $O(n)$-approximation of $d^{*}=$ $\pi(s, t)$, i.e., it returns a value $\tilde{d}$ such that $d^{*} \leq \tilde{d} \leq c n d^{*}$ for some constant $c>0$. By augmenting $\tilde{\mathcal{V}}$ with a linear number of additional edges, each a constant-clearance path between two points on the boundary of a cell of $\tilde{\mathcal{V}}$, the algorithm constructs a graph $G_{1}$ with $O(n)$ vertices and edges and computes a minimum-cost path from the source $s$ to the target $t$ in $G_{1}$.

Equipped with the value $\tilde{d}$, the second stage computes an $O(1)$-approximation of $d^{*}$. For a given $d \geq 0$, this algorithm constructs a graph $G_{2}=G_{2}[d]$ by sampling $O(n)$ points on the boundary of each cell $T$ of $\tilde{\mathcal{V}}$ and connecting these sample points by adding $O(n)$ edges (besides the boundary of $T$ ), each of which is again a constant-clearance path. The resulting graph $G_{2}$ is planar and has $O\left(n^{2}\right)$ edges total, so a minimum-cost $s, t$-path in $G_{2}$ can be computed in $O\left(n^{2}\right)$ time [12]. We show that if $d \geq d^{*}$, then the cost of the optimal $s, t$-path in $G_{2}$ is $O(d)$. Therefore, if $d \in\left[d^{*}, 2 d^{*}\right]$, the cost of the optimal path is $O\left(d^{*}\right)$. Using the value of $\tilde{d}$, we run the above procedure for $O(\log n)$ different values of $d$, namely $d \in\left\{\tilde{d} / 2^{i} \mid 0 \leq i \leq\left\lceil\log _{2} c n\right\rceil\right\}$, and return the least costly path among them. Let $\hat{d}$ be the cost of the path returned. 


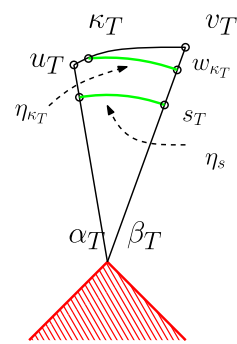

(a) Point-obstacle cell

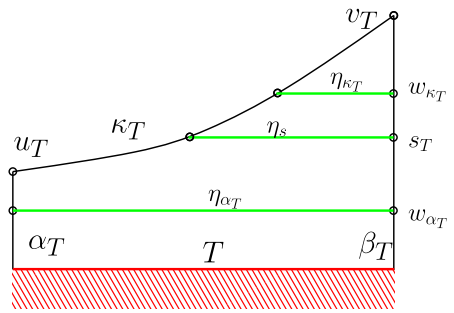

(b) Line-obstacle cell

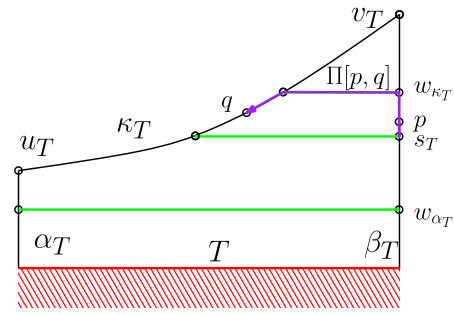

(c) $(p, q)$-path $\gamma$

Fig. 7. (a), (b) Edges added within cell $T$ for the $O(n)$-approximation algorithm. (c) Visualization of proof of Lemma 4.1.

Finally, using the value $\hat{d}$, the third stage samples $O(n / \varepsilon)$ points on the boundary of each cell $T$ of $\tilde{\mathcal{V}}$ and connects each point to $O((1 / \varepsilon) \log (n / \varepsilon))$ other points on the boundary of $T$ by an edge. Unlike the last two stages, each edge is no longer a constant-clearance path but it is a minimum-cost path between its endpoints lying inside $T$. The resulting graph $G_{3}$ has $O\left(n^{2} / \varepsilon\right)$ vertices and $O\left(\left(n^{2} / \varepsilon^{2}\right) \log (n / \varepsilon)\right)$ edges. The overall algorithm returns the minimum-cost path in $G_{3}$. Anchor points and well-behaved paths play a pivotal role in each stage of the algorithm.

\subsection{Computing an $O(n)$-approximation Algorithm}

Here, we describe a near-linear time algorithm to obtain an $O(n)$-approximation of $d^{*}$. We augment $\tilde{\mathcal{V}}$ with $O(n)$ additional edges as described below to create the graph $G_{1}=\left(V_{1}, E_{1}\right)$.

We do the following for each cell $T$ of $\tilde{\mathcal{V}}$. We compute anchor points $w_{\alpha_{T}}$ and $w_{\kappa_{T}}$ as described in Lemma 3.3. Let $s_{T}$ be the point on $\beta_{T}$ of clearance $\min \left\{\operatorname{clr}\left(v_{T}\right), \operatorname{clr}(s)\right\}$ (it is worth noting that we only need to consider the source $s$ and not the target $t$ since we assume that $\operatorname{clr}(s) \leq \operatorname{clr}(t))$. Set $W_{T}=\left\{w_{\alpha_{T}}, w_{\kappa_{T}}, s_{T}\right\}, E_{T}=\left\{\eta_{w} \mid w \in W_{T}\right\}$, and $\bar{W}_{T}=\left\{\bar{w}_{\alpha_{T}}, \bar{w}_{\kappa_{T}}, \bar{s}_{T}\right\}$. Vertex set $V_{1}$ is the set of Voronoi vertices plus the set $W_{T} \cup \bar{W}_{T}$ for all cells $T \in \tilde{\mathcal{V}}^{3}$. Next, for each edge $e$ of $\tilde{\mathcal{V}}$, we add the portion of $e$ between two consecutive vertices of $V_{1}$ as an edge of $E_{1}$, and for each cell $T \in \tilde{V}$ we also add $E_{T}$ to $E_{1}$. See Figure 7(a) and (b). (Note that if $s_{T}=v_{T}$ then $\eta_{s}$ is a trivial path and there is no need to add $\eta_{s}$ to $E_{1}$. Paths $\eta_{w_{\alpha_{T}}}$ and $\eta_{w_{\kappa_{T}}}$ may be trivial as well.) The cost $\mu(e)$ for each edge $e \in E_{1}$ is computed using Equation (1) or the equations of Wein et al. [20] for Voronoi edges. By construction, $\left|V_{1}\right|=O(n)$ and $\left|E_{1}\right|=O(n)$. We compute and return, in $O(n \log n)$ time, an optimal $s, t$-path in $G_{1}$.

Lemma 4.1. Graph $G_{1}$ contains an $s, t$-path of cost at most $O(n) \cdot d^{*}$.

Proof. Recall, $\Pi^{*}$ is an optimal $s, t$-path. We will deform $\Pi^{*}$ into another $s, t$-path $\tilde{\Pi}$ of cost $O(n) \cdot d^{*}$ that enters or exits the interior of a cell of $\tilde{\mathcal{V}}$ only at the vertices of $V_{1}$ and follows an arc of $E_{T}$ in the interior of the cell $T$. By construction, $\tilde{\Pi}$ will be a path in $G_{1}$, which will imply the claim.

By construction $s, t \in V_{1}$. Let $\Pi$ denote the current path that we have obtained by deforming $\Pi^{*}$. Let $T \in \tilde{\mathcal{V}}$ be the first cell (along $\Pi$ ) such that $\Pi$ enters the interior of $T$ but $\operatorname{int}(T) \cap \Pi$ is not an arc of $E_{T}$. Let $p$ (resp. q) be the first (resp. last) point of $\Pi \cap T$. Recall, $G_{1}$ contains subdivisions of every

\footnotetext{
${ }^{3}$ Note that as we consider each cell independently, we actually consider each edge $e$ twice as it is adjacent to two cells and add vertices on $e$ for each cell independently. The set of vertices put on $e$ is the union of these two sets. Considering each edge twice does not change the complexity of the algorithm or its analysis, and doing so simplifies the algorithm's description.
} 


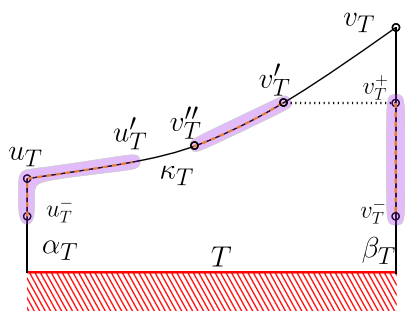

Fig. 8. Samples placed on the edges of a cell $T$ of $\tilde{\mathcal{V}}$. The sampled regions are depicted in purple. For the constant-factor approximation algorithm, samples are placed on $\beta_{T}$ only.

edge in $\tilde{\mathcal{V}}$. If both $p$ and $q$ lie on the same edge of $T$ or neither of them lies on $\beta_{T}$, we replace $\Pi[p, q]$ with the well-behaved path $\gamma(p, q)$, because $\gamma(p, q) \subseteq \partial T$ in this case. Suppose $p \in \beta_{T}$ and $q \notin \beta_{T}$ (the other case is symmetric). We replace $\Pi[p, q]$ with $\Pi[p, q]=p^{\sim} s_{T} \circ \gamma\left(s_{T}, q\right)$, i.e., the segment $\tilde{p} s_{T} \subseteq \beta_{T}$ followed by the well-behaved path $\gamma\left(s_{T}, q\right)$. By Lemma 3.3, $\operatorname{cl}\left(\operatorname{int}(T) \cap \gamma\left(s_{T}, q\right)\right) \in E_{T}$.

We repeat the above step until no such cell $T$ is left. Since the above step is performed at most once for each cell of $\tilde{\mathcal{V}}$, we obtain the final path $\tilde{\Pi}$ in $O(n)$ steps.

We now bound the cost of $\tilde{\Pi}$. If $\Pi[p, q]$ is replaced by $\gamma(p, q)$, then by Lemma $3.1, \mu(\gamma(p, q)) \leq$ $3 \mu(\Pi[p, q])$. On the other hand, if $p \in \beta_{T}$ and $q \notin \beta_{T}$, then $\mu(\tilde{\Pi}[p, q])=\mu\left(p^{\sim} s_{T}\right)+\mu\left(\gamma\left(s_{T}, q\right)\right)$. By the triangle inequality, $\pi\left(s_{T}, q\right) \leq \pi\left(s_{T}, p\right)+\pi(p, q)$, and by Lemma 3.2 ,

$$
\mu\left(\gamma\left(s_{T}, q\right)\right) \leq 11 \pi\left(s_{T}, q\right) \leq 11\left(\pi\left(s_{T}, p\right)+\pi(p, q)\right) .
$$

By Lemma 2.2, $\pi\left(p, s_{T}\right)=\mu\left(\tilde{p}^{\sim} s_{T}\right) \leq d^{*}$. Putting everything together,

$$
\begin{aligned}
\mu(\tilde{\Pi}[p, q]) & \leq 12 \pi\left(p, s_{T}\right)+11 \pi(p, q) \\
& \leq 12 d^{*}+11 \pi(p, q)=O\left(d^{*}\right) .
\end{aligned}
$$

Summing over all $O(n)$ steps, the cost of $\tilde{\Pi}$ is $O(n) \cdot d^{*}$.

We thus obtain the following.

THEOREM 4.2. Let $\mathcal{O}$ be a set of disjoint polygonal obstacles in the plane with $n$ vertices total, and let $s, t$ be two points outside $\mathcal{O}$. There exists an $O(n \log n)$-time $O(n)$-approximation algorithm for computing the minimal-cost path between $s$ and $t$.

\subsection{Computing a Constant-Factor Approximation}

Recall that, given an estimate $d$ of the cost $d^{*}$ of the optimal path, we construct a planar graph $G_{2}=$ $G_{2}[d]$ by sampling points along the edges of the refined Voronoi diagram $\tilde{\mathcal{V}}$. The sampling procedure here can be thought of as a warm-up for the more-involved sampling procedure given in Section 4.3.

Let $T$ be a Voronoi cell of $\tilde{\mathcal{V}}$. Let $v_{T}^{-}$and $v_{T}^{+}$be the points on $\beta_{T}$ with clearance $\min \left\{\operatorname{clr}\left(v_{T}\right), \operatorname{clr}(t) / \exp (d)\right\}$ and $\min \left\{\operatorname{clr}\left(v_{T}\right), \operatorname{clr}(s) \cdot \exp (d)\right\}$, respectively. We refer to the segment $\hat{\beta}_{T}=v_{T}^{-} v_{T}^{+} \subseteq \beta_{T}$ as the marked portion of $\beta_{T}$. By $(4), \mu\left(\hat{\beta}_{T}\right)=O(d)$. We place sample points on $\hat{\beta}_{T}$, its endpoints always being sampled, so that the cost between consecutive samples is exactly $\frac{d}{n}$ (except possibly at one endpoint). Given a sample point $p$ on an edge of $\tilde{\mathcal{V}}$, it is straightforward to compute the coordinates of the sample point $p^{\prime}$ on the same edge such that $\pi\left(p, p^{\prime}\right)=c$ for any $c>0$. Simply use the formula for the cost along a Voronoi edge given in [20, Corollary 8]. We emphasize that the points are separated evenly by cost; the samples are not uniformly placed by Euclidean distance along the edge; see Figure 8. 
For each cell $T \in \tilde{\mathcal{V}}$, let $W_{T}$ be the set of sample points on $\beta_{T}$ plus the anchor points $w_{\kappa_{T}}$ and $w_{\alpha_{T}}$. For each point $w \in W_{T}$, we compute the constant-clearance arc $\eta_{w}$. Let $E_{T}=\left\{\eta_{w} \mid w \in W_{T}\right\}$ and $\bar{W}_{T}=\left\{\bar{w} \mid w \in W_{T}\right\}$ be the set of other endpoints of arcs in $E_{T}$. Set $V_{2}$ is the set of vertices of $\tilde{\mathcal{V}}$ plus the set $W_{T} \cup \bar{W}_{T}$ over all cells in $\tilde{\mathcal{V}}$. For each edge of $\tilde{\mathcal{V}}$, we add the portions between consecutive sample vertices of $V_{2}$ to $E_{2}$, and we also add $E_{T}$, over all cells $T \in \tilde{\mathcal{V}}$, to $E_{2}$. The cost of each edge in $E_{2}$ is computed as before. We have $\left|V_{2}\right|,\left|E_{2}\right|=O\left(n^{2}\right)$, and $G_{2}$ can be constructed in $O\left(n^{2}\right)$ time.

The refined Voronoi diagram $\tilde{\mathcal{V}}$ is planar. Every edge $\eta_{w}$ added to create $G_{2}$ stays within a single cell of $\tilde{\mathcal{V}}$ and has constant clearance. Therefore, no new crossings are created during its construction, and $G_{2}$ is planar as well. We compute the minimum-cost $s, t$-path in $G_{2}$, in $O\left(n^{2}\right)$ time, using the algorithm of Henzinger et al. [12].

Lemma 4.3. For an optimal $s-t$ path $\Pi^{*}$, if $d \geq d^{*}$, then $\Pi^{*} \cap \beta_{T} \subseteq \hat{\beta}_{T}$ for any cell $T \in \tilde{\mathcal{V}}$.

Proof. Let $p_{\min }$ be the point where $\Pi^{*}$ attains the minimum clearance. Clearly, $\pi(s, t)=$ $\pi\left(s, p_{\min }\right)+\pi\left(p_{\min }, t\right)$. Using this observation together with Lemma 2.1(i) and the assumption that $\operatorname{clr}(s) \leq \operatorname{clr}(t)$, we conclude that the clearance of any point on $\Pi^{*}$ is at least $\operatorname{clr}(t) / \exp \left(d^{*}\right) \geq$ $\operatorname{clr}(t) / \exp (d)$. A similar argument implies the clearance of any point on $\Pi^{*}$ is at most $\operatorname{clr}(s)$. $\exp (d)$. Hence, $\Pi^{*} \cap \beta_{T} \subseteq \hat{\beta}_{T}$.

LEMMA 4.4. For $d \geq d^{*}$, graph $G_{2}[d]$ contains an $s, t$-path of cost $O(d)$.

Proof. We deform the optimal path $\Pi^{*}$ into a path $\tilde{\Pi}$ of $G_{2}$ in the same way as in the proof of Lemma 4.1 except for the following twist. As in Lemma 4.1, let $p$ (resp. $q$ ) be the first (resp. last) point on $\Pi^{*}$ in a cell $T$ of $\tilde{\mathcal{V}}$. If $p \in \beta_{T}$ and $q \notin \beta_{T}$, let $p^{\prime}$ be a sample point on $\beta_{T}$ such that $\pi\left(p, p^{\prime}\right) \leq d / n$; the existence of $p^{\prime}$ follows from Lemma 4.3. We replace $\Pi^{*}[p, q]$ with $\tilde{\Pi}_{T}=p p^{\prime} \circ \gamma\left(p^{\prime}, q\right)$, i.e., $p^{\prime}$ replaces $w_{s}$ in the proof of Lemma 4.1. Since $\pi\left(p, p^{\prime}\right) \leq d / n$, we have

$$
\mu\left(\tilde{\Pi}_{T}\right) \leq 11 \pi(p, q)+O(d / n)
$$

Summing over all steps in the deformation of $\Pi^{*}$ and using the fact $d \geq d^{*}=\mu\left(\Pi^{*}\right)$, we obtain $\mu(\tilde{\Pi})=O(d)$. It is clear from the construction that $\tilde{\Pi}$ is a path in $G_{2}$.

For our constant-factor approximation algorithm, we perform an exponential search over the values of path costs. Let $\tilde{d} \leq c n d^{*}$ be the cost of the path returned by the $O(n)$-approximation algorithm (Section 4.1). For each $i$ from 0 to $\lceil\log c n\rceil$, we choose $d_{i}=\tilde{d} / 2^{i}$. We run the above procedure to construct a graph $G_{2}\left[d_{i}\right]$ and compute a minimum-cost path $\Pi_{i}$ in the graph. We compute $k=\operatorname{argmin}_{i} \mu\left(\Pi_{i}\right)$ and return $\Pi_{k}$.

Fix integer $\hat{i}$ so $d^{*} \leq d_{\hat{i}} \leq 2 d^{*}$. By Lemma 4.4, we have

$$
\mu\left(\Pi_{i}\right) \leq \mu\left(\Pi_{\hat{i}}\right)=O\left(d_{\hat{i}}\right)=O\left(d^{*}\right) .
$$

THEOREM 4.5. Let $\mathcal{O}$ be a set of disjoint polygonal obstacles in the plane with $n$ vertices total, and let $s, t$ be two points outside $\mathcal{O}$. There exists an $O\left(n^{2} \log n\right)$ time $O(1)$-approximation algorithm for computing the minimum-cost path between $s$ and $t$.

\subsection{Computing the Final Approximation}

Finally, let $d$ be the estimate returned by our constant factor approximation algorithm so that $d^{*} \leq d \leq c d^{*}$ for some constant $c$. We construct a graph $G_{3}=\left(V_{3}, E_{3}\right)$ by sampling $O(n / \varepsilon)$ points along each edge of $\tilde{\mathcal{V}}$ and connecting (a certain choice of) $O\left(\frac{n}{\varepsilon^{2}} \log \frac{n}{\varepsilon}\right)$ pairs of sample points on the boundary of each cell of $\tilde{\mathcal{V}}$ by "locally optimal" paths. We guarantee $\left|V_{3}\right|=O\left(\frac{n^{2}}{\varepsilon}\right)$ 


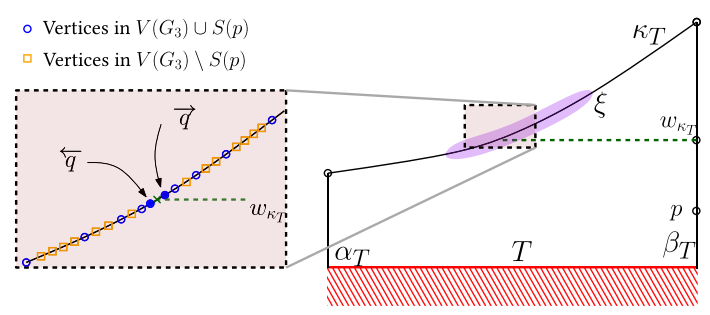

Fig. 9. Vertices in $S(p)$ that are used to construct the set of edges of $G_{3}$.

and $\left|E_{3}\right|=O\left(\frac{n^{2}}{\varepsilon^{2}} \log \frac{n}{\varepsilon}\right)$. We compute and return, in $O\left(\frac{n^{2}}{\varepsilon^{2}} \log \frac{n}{\varepsilon}\right)$ time, a minimum-cost path in $G_{3}[10]$.

Vertices of $G_{3}$. Let $\underline{c}=\operatorname{clr}(t) / \exp (d)$ and $\bar{c}=\operatorname{clr}(s) \cdot \exp (d)$. Let $T$ be a cell of $\tilde{\mathcal{V}}$. For each edge of $T$, we mark at most two connected portions, each of cost $O(d)$. We refer to each marked portion as an edgelet. We sample points on each edgelet so that two consecutive samples lie at cost $\varepsilon d / n$ apart; endpoints of each edgelet are always included in the sample. The total number of samples placed on $\partial T$ is $O(n / \varepsilon)$. We now describe the edgelets of $T$.

Let $u_{T}^{-}, u_{T}^{+}$be points on $\alpha_{T}$ of clearance $\min \left\{\operatorname{clr}\left(u_{T}\right), \underline{c}\right\}$ and $\min \left\{\operatorname{clr}\left(u_{T}\right), \bar{c}\right\}$, respectively. Similarly, let $v_{T}^{-}, v_{T}^{+}$be points on $\beta_{T}$ of clearance $\min \left\{\operatorname{clr}\left(v_{T}\right), \underline{c}\right\}$ and $\min \left\{\operatorname{clr}\left(v_{T}\right), \bar{c}\right\}$, respectively. The edgelets on $\alpha_{T}$ and $\beta_{T}$ are the segments $u_{T}^{-} u_{T}^{+}$and $v_{T}^{-} v_{T}^{+}$, respectively. Next, we mark (at most) two edgelets on $\kappa_{T}$ : If $\mu\left(\kappa_{T}\right) \leq 2 d$, then the entirety of $\kappa_{T}$ is a single edgelet; otherwise, let $u_{T}^{\prime} \in \kappa_{T}$ be the point such that $\mu\left(\kappa_{T}\left[u_{T}, u_{T}^{\prime}\right]\right)=2 d$. Let $v_{T}^{\prime} \in \kappa_{T}$ be the point of clearance $\operatorname{clr}\left(v_{T}^{+}\right)$. If $\mu\left(\kappa_{T}\left[u_{T}^{\prime}, v_{T}^{\prime}\right]\right) \leq 4 d$, then $\kappa_{T}\left[u_{T}, v_{T}^{\prime}\right]$ is the only edgelet on $\kappa_{T}$. Otherwise, let $v_{T}^{\prime \prime} \in \kappa_{T}$ be the point such that $\operatorname{clr}\left(v_{T}^{\prime \prime}\right) \leq \operatorname{clr}\left(v_{T}^{\prime}\right)$ and $\mu\left(\kappa_{T}\left[v_{T}^{\prime}, v_{T}^{\prime \prime}\right]\right)=4 d ; \kappa_{T}$ has two edgelets $\kappa_{T}\left[u_{T}, u_{T}^{\prime}\right]$ and $\kappa_{T}\left[v_{T}^{\prime}, v_{T}^{\prime \prime}\right]$. See Figure 8. We repeat this procedure for all cells of $T$. Set $V_{3}$ is the set of all samples placed on the edges of $\tilde{\mathcal{V}}$. We have $\left|V_{3}\right|=O\left(n^{2} / \varepsilon\right)$.

The Edges of $G_{3}$. Let $T$ be a cell of $\tilde{\mathcal{V}}$ incident to obstacle feature $o$. We say two points $p$ and $q$ in $T$ are locally reachable from one another if the minimum-cost $p, q$-path relative only to $o$ lies within $T$. Equivalently, the minimum-cost path relative to $o$ is equal to the minimum-cost path relative to $\mathcal{O}$.

Let $p \in \partial T$ be a sample point. We compute a subset $S(p) \subseteq V_{3}$ of candidate neighbors of $p$ in $G_{3}$. Let $N(p) \subseteq S(p)$ be the subset of these points that are locally reachable from $p$. We connect $p$ to each point $q \in N(p)$ by an edge in $E_{3}$ of cost $\pi(p, q)$. By definition, the minimum-cost path between $p$ and $q$ lies inside $T$. Finally, as in $G_{1}$ and $G_{2}$, we add the portion of each edge of $\tilde{\mathcal{V}}$ between two sample points as an edge of $E_{3}$.

We now describe how we construct $S(p)$. Let $\xi$ be an edgelet of $\partial T$ such that $p$ and $\xi$ do not lie on the same edge of $T$. We first define a shadow point $\breve{p}$ of $p$. If $p \in \alpha_{T} \cup \kappa_{T}$, then $\breve{p}=p$. If $p \in \beta_{T}$, and $\xi \in \kappa_{T}\left(\right.$ resp. $\left.\xi \in \alpha_{T}\right)$, then $\breve{p}=p$ if $\operatorname{clr}(p) \geq \operatorname{clr}\left(w_{\kappa_{T}}\right)\left(\right.$ resp. $\left.\operatorname{clr}(p) \geq \operatorname{clr}\left(w_{\alpha_{T}}\right)\right)$, and $\breve{p}=w_{\kappa_{T}}$ (resp. $w_{\alpha_{T}}$ ) otherwise. Let $\overleftarrow{q}$ (resp. $\vec{q}$ ) be the sample point on $\xi$ of highest (resp. lowest) clearance less (resp. more) than $\breve{p}$, if such a point exists. Exactly one of $\overleftarrow{q}$ or $\vec{q}$ may not exist if no point of clearance $\breve{p}$ exists on $\xi$; in this case, the construction implies that $\overleftarrow{q}$ is the endpoint of $\xi$ of higher clearance or $\vec{q}$ is the endpoint of $\xi$ of lower clearance. If $\overleftarrow{q}$ exists, we add $\overleftarrow{q}$ to $S(p)$. We iteratively walk along sample points of $\xi$ in decreasing order of clearance starting with the first sample point encountered after $\stackrel{\leftarrow}{q}$. For each non-negative integer $i$, we add the point $q_{i}$ encountered at step $\left\lfloor(1+\varepsilon)^{i}\right\rfloor$ of the walk until we reach an endpoint of $\xi$. Similarly, if $\vec{q}$ exists, we add to $S(p)$ the point $\vec{q}$ and perform the walk along points of greater clearance. See Figure 9 . Finally, we add the two endpoints of $\xi$ to $S(p)$. We repeat this step for all edgelets on $\partial T$. Since $\xi$ has $O(n / \varepsilon)$ sample 
points and $\partial T$ has at most four edgelets, $|S(p)|=O((1 / \varepsilon) \log (n / \varepsilon))$, and $S(p)$ can be constructed in $O(|S(p)|)$ time.

Analysis. It is clear from the construction that $\left|V_{3}\right|=O\left(\frac{n^{2}}{\varepsilon}\right),\left|E_{3}\right|=O\left(\frac{n^{2}}{\varepsilon^{2}} \log \frac{n}{\varepsilon}\right)$, and that $G_{3}$ can be constructed in time $O\left(\frac{n^{2}}{\varepsilon^{2}} \log \frac{n}{\varepsilon}\right)$. By using Dijkstra's algorithm with Fibonacci heaps [10], a minimum-cost path in $G_{3}$ can be computed in $O\left(\frac{n^{2}}{\varepsilon^{2}} \log \frac{n}{\varepsilon}\right)$ time. So it remains to prove that the algorithm returns a path of cost at most $(1+O(\varepsilon)) \pi(s, t)$. By rescaling $\varepsilon$, we can thus compute an $s, t$ - path of cost at most $(1+\varepsilon) \pi(s, t)$ in time $O\left(\frac{n^{2}}{\varepsilon^{2}} \log \frac{n}{\varepsilon}\right)$.

Lemma 4.6. Let $\Pi^{*}$ be a minimum-cost $s, t$-path. For every edge e $\in \tilde{\mathcal{V}}, \Pi^{*} \cap$ e lies inside the marked portion of e.

Proof. Fix an edge $e \in \tilde{\mathcal{V}}$, and let $q \in \Pi^{*} \cap e$. We aim to prove that $q$ lies inside the marked portion of $e$. Recall that $d \geq d^{*}$. The proof of Lemma 4.3 already handles the case of $e$ being an internal edge.

Now, suppose $e$ is an external edge. We assume $\mu(e)>2 d$; otherwise, the proof is trivial. We have $e=\kappa_{T}$ and $e=\kappa_{T^{\prime}}$ for two adjacent Voronoi cells $T$ and $T^{\prime}$. By construction, point $s$ lies outside the interior of $T \cup T^{\prime}$. Therefore, $\Pi^{*}[s, q]$ intersects at least one internal edge incident to $T$ or $T^{\prime}$ at some point $p$. Without loss of generality, assume that internal edge belongs to $T$. We have two cases.

Case 1: $p \in \alpha_{T}$. Since $\operatorname{clr}(p) \leq \operatorname{clr}\left(u_{T}\right) \leq \operatorname{clr}(q)$, we have $\pi\left(p, u_{T}\right) \leq d^{*}$. By triangle inequality,

$$
\pi\left(u_{T}, q\right) \leq \pi\left(u_{T}, p\right)+\pi(p, q) \leq 2 d^{*} \leq 2 d .
$$

Case 2: $p \in \beta_{T}$. By Equation (4), $\pi\left(v_{T}^{+}, p\right) \leq 2 d$. Recall from the proof of Lemma 4.3 that the clearance of any point on $\Pi^{*}$ is at most $\operatorname{clr}(s) \cdot \exp (d)$. We defined $\eta_{v_{T}^{+}}$as the line segment or circular arc with $v_{T}^{+}$as one of its endpoints; $v_{T}^{\prime}$ is its other endpoint. One can easily verify $\mu\left(\eta_{v_{T}^{+}}\right) \leq d^{*}$; see the last paragraph of the proof of Lemma 3.2. By the triangle inequality,

$$
\pi\left(v_{T}^{\prime}, q\right) \leq \mu\left(\eta_{v_{T}^{+}}\right)+\pi\left(v_{T}^{+}, p\right)+\pi(p, q) \leq 2 d^{*}+2 d \leq 4 d .
$$

Now, we prove a property of locally reachable points from a fixed point that will be crucial for our analysis.

LEMmA 4.7. Let $T$ be a cell of $\tilde{\mathcal{V}}$, and let $p \in \partial T$. For every edge e of $T$, the set of points on e locally reachable from $p$, if non-empty, is a connected portion of $e$ and contains an endpoint of $e$.

Proof. Let $o$ be the feature of $\mathcal{O}$ associated with $T$. We consider two main cases.

Case 1: $o$ is a vertex. Without loss of generality, $o$ lies at the origin, edge $\alpha_{T}$ intersects the line $y=0$ at the origin with angle $\theta_{\alpha}$, edge $\beta_{T}$ intersects the line $y=0$ at the origin with angle $\theta_{\beta}$, and $\theta_{\beta}>\theta_{\alpha} \geq 0$. We consider a map $f: \mathbb{R}^{2} \rightarrow \mathbb{R}^{2}$ taking points to what we refer to as the transformed plane. Given in polar coordinates point $(r, \theta)$, the map $f$ is defined as $f(r, \theta)=(\theta, \ln r)$. For a point $x \in \mathbb{R}^{2}$, let $x^{*}=f(x)$, and for a point set $X \subseteq \mathbb{R}^{2}$, let $X^{*}=\{f(x) \mid x \in X\}$. Both $\alpha_{T}$ and $\beta_{T}$ become vertical rays in the transformed plane going to $-\infty$. Further, it is straightforward to show that $\kappa_{T}$ becomes a convex curve in the transformed plane when restricted to values of $\theta$ such that $\theta_{\alpha} \leq \theta \leq \theta_{\beta}$. Therefore, $T^{*}$ is a semi-bounded pseduo-trapezoid. By (P1) in Section 2 (see also Reference [20]), the minimum-cost path with respect to $o$ between two points $a, b \in T$ maps to the line segment $a^{*} b^{*}$ (see Figure 10). So $a$ and $b$ are locally reachable if $a^{*} b^{*} \subseteq T^{*}$, i.e., $a^{*}$ and $b^{*}$ are visible from each other (see Figure 11). 


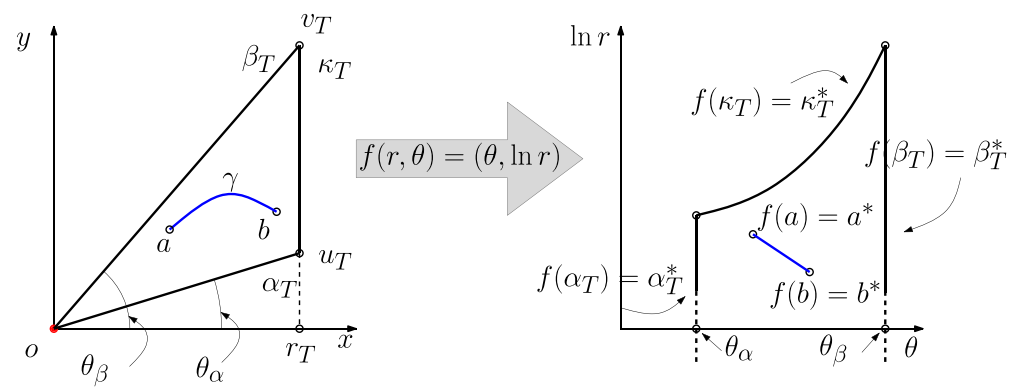

Fig. 10. Case 1 of proof of Lemma 4.7: a cell of a point obstacle in $\tilde{\mathcal{V}}$ and the optimal path between points $a$ and $b$ (blue) in the original (left) and transformed plane (right), respectively.

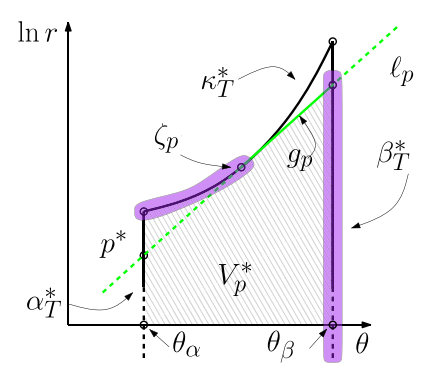

(a) $p \in \alpha_{T}$

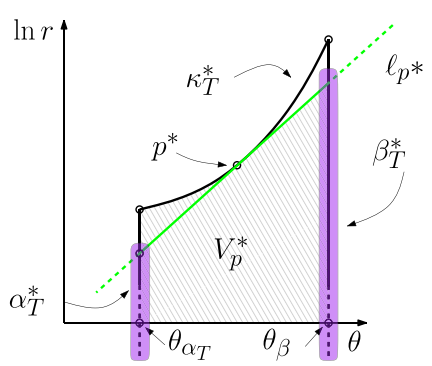

(b) $p \in \kappa_{T}$

Fig. 11. Case 1 of proof of Lemma 4.7: the set of locally reachable points (purple) from the point $p$ in the transformed plane; the solid part of $\ell_{p}$ is $g_{p}$.

For a point $p^{*} \in(\partial T)^{*}$, let $V_{p}^{*} \subseteq T^{*}$ be the set of points of $T^{*}$ visible from $p^{*}, \ell_{p}$ the line tangent to $\kappa_{T}^{*}$ from $p^{*}$ (if it exists; if no such tangent exists then the proof is trivial), and $\zeta_{p}=\ell_{p} \cap \kappa_{T}^{*}$. Note that $\ell_{p}$ is well defined, because either $p^{*} \in \kappa_{T}^{*}$ or the $x$-monotone convex curve $\kappa_{T}^{*}$ either lies to the left or to the right of $p^{*}$. The closure of $\partial V_{p}^{*} \backslash(\partial T)^{*}$ consists of a line segment $g_{p}=a^{*} b^{*} \subset \ell_{p}$. If $p \notin \kappa_{T}$, then $\zeta_{p}$ is one endpoint of $g_{p}$ and the other endpoint lies on $\alpha_{T}^{*}$ or $\beta_{T}^{*}$. In either case, for any edge $e \in \partial T$, if $\left(e^{*} \backslash\left\{p^{*}\right\}\right) \cap V_{p}^{*} \neq \emptyset$, then it is a connected arc and contains one of the endpoints of $e^{*}$, as claimed.

Case 2: $o$ is an edge. Without loss of generality, $o$ lies on the line $y=0$, the edge $\alpha_{T}$ lies on the line $x=x_{\alpha}$, the edge $\beta_{T}$ lies on the line $x=x_{\beta}$, and $x_{\beta}>x_{\alpha} \geq 0$. There is no equally convenient notion of the transformed plane for edge feature $o$, but we are still able to use similar arguments to those given in Case 1.

In this case, for two points $a, b \in T$, the minimum-cost path with respect to $o$ from $a$ to $b$ is the circular arc with $a$ and $b$ as its endpoints and centered at the $x$-axis (see (P2) in Section 2). Therefore, $a$ and $b$ are locally reachable if this circular arc does not cross $\kappa_{T}$.

Fix a point $p=\left(x_{p}, y_{p}\right) \in \partial T$. If $p \in \alpha_{t} \cup \beta_{T}$, then all points on the edge of $T$ containing $p$ are locally reachable, and if $p \in \kappa_{T}$ then no point on $\kappa_{T} \backslash\{p\}$ is locally reachable from $p$. So we will focus on edges of $T$ that do not contain $p$.

Let $\mathcal{C}_{p}$ denote the one-parameter family of circles that pass through $p$ and are centered at the $x$-axis. For any $q \in T \backslash\{p\}$, there is a unique circle $C_{q} \in \mathcal{C}_{p}$ that passes through $q$. We parameterize the circles in $\mathcal{C}_{p}$ with the $x$-coordinate of its center, i.e., $C(t) \in \mathcal{C}_{p}$ for $t \in(-\infty, \infty)$ and is centered at $(t, 0)$. Let $C^{+}(t)$ (resp. $\left.C^{-}(t)\right)$ be the circular arc of $C(t)$ 


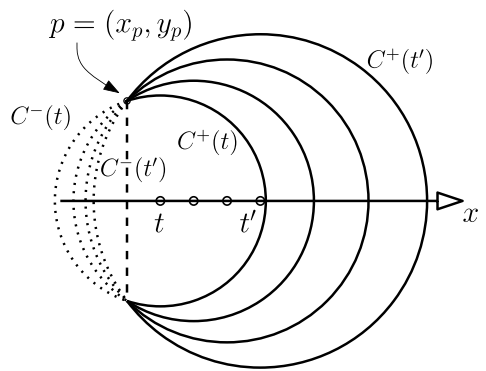

(a)

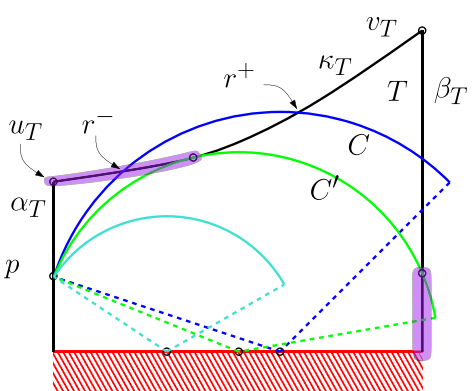

(b)

Fig. 12. Illustration of properties of $\bigodot_{p}$ in Case 2 of proof of Lemma 4.7.

lying to the right (resp. left) of the line $x=x_{p}$. See Figure 12(a). The following properties of $\mathcal{C}_{p}$ are easily verified:

(a) For $t<t^{\prime}, C^{+}(t)$ (resp. $\left.C^{-}\left(t^{\prime}\right)\right)$ lies inside of $C\left(t^{\prime}\right)$ (resp. $\left.C(t)\right)$; see Figure 12(a).

(b) If a circle $C \in \mathcal{C}_{p}$ intersects $\kappa_{T}$ at two points, say, $r^{-}$and $r^{+}$, then there is another circle $C^{\prime} \in \mathcal{C}_{p}$ that is tangent to $\kappa_{T}$ between $r^{-}$and $r^{+}$; see Figure $12(\mathrm{~b})$.

(c) A circle in $\mathcal{C}_{p}$ intersects $\alpha_{T}$ or $\beta_{T}$ in at most one point.

(d) There is at most one circle $C \in \mathcal{C}_{p}$ that is tangent to $\kappa_{T}$.

Properties (a) and (b) are straightforward; (c) follows from (a) and a continuity argument; (d) follows from (a), (c), and the convexity of $\kappa_{T}$.

If there is no circle in $\mathcal{C}_{p}$ that is tangent to $\kappa_{T}$ then for any point $q \in T$, the $\operatorname{arc} C_{q}[p, q]$ lies inside $T$, so every point in $T$ is locally reachable, and the lemma follows.

Next, assume there is a circle $C_{0} \in \mathcal{C}_{p}$ that is tangent to $\kappa_{T}$ at a point $r_{p}$. By (d), $C_{0}$ is the only such circle. There are three cases:

(i) If $p \notin \alpha_{T}$, then points lying on $\alpha_{T}$ that are inside $C_{0}$ are locally reachable from $p$ by property (a).

(ii) Similarly, if $p \notin \beta_{T}$, then the points in $\operatorname{int}(C) \cap \beta_{T}$ are locally reachable, again by property (a).

(iii) If $p \in \alpha_{T}$ (resp. $p \in \beta_{T}$ ), then the points in $\kappa_{T}\left[u_{t}, r_{p}\right]$ (resp. $\kappa_{T}\left[r_{p}, v_{T}\right]$ ) are locally reachable from $p$ by properties (c) and (d).

Hence, in each case at most one connected portion of an edge $e$ of $T$ is locally reachable from $p$, and it contains one endpoint of $e$.

Lemma 4.8. Graph $G_{3}$ contains an $s, t$-path of cost at most $(1+O(\varepsilon)) d^{*}$.

Proof. Once again, we deform the optimal path $\Pi^{*}$ into a path $\tilde{\Pi}$ of $G_{3}$ as in Lemmas 4.1 and 4.4. Let $\Pi$ denote the current path that we have obtained by deforming $\Pi^{*}$. Let $T \in \tilde{\mathcal{V}}$ be the first cell such that $\Pi$ enters $\operatorname{int}(T)$ but $\operatorname{int}(T) \cap \Pi$ is not an $\operatorname{arc}$ of $E_{3}$. Let $p \in \Pi$ be the first point (on $\partial T$ ) at which $\Pi$ enters in $\operatorname{int}(T)$, and let $q$ be the next point on $\Pi \cap \partial T$, i.e., $\operatorname{int}(\Pi[p, q]) \subset \operatorname{int}(T)$. If both $p$ and $q$ lie on the same edge $e$ of $T$, we replace $\Pi^{*}[p, q]$ with the portion of $e$ between $p$ and $q$, denoted by $\tilde{\Pi}_{T}$; note that $\mu\left(\tilde{\Pi}_{T}\right)=\pi(p, q)$.

Now, suppose $p \in \beta_{T}$ and $q \in \kappa_{T}$. The other cases are similar. Points $p$ and $q$ are locally reachable from each other. By Lemmas 4.6 and 4.7, there exists a sample point $p^{\prime}$ locally reachable from $q$ on $\beta_{T}$ such that $\pi\left(p, p^{\prime}\right) \leq \varepsilon d / n$. We have $\pi\left(p^{\prime}, q\right) \leq \pi(p, q)+\varepsilon d / n$. Suppose there exists a point $q^{\prime} \in S\left(p^{\prime}\right)$ on $\kappa_{T}$ locally reachable from $p^{\prime}$ such that $\pi\left(q, q^{\prime}\right) \leq \varepsilon d / n$. Let $a$ be the minimumcost path from $p^{\prime}$ to $q^{\prime}$. In this case, we replace $\Pi^{*}[p, q]$ with $\tilde{\Pi}_{T}=p p^{\prime} \circ a \circ \gamma\left(q^{\prime}, q\right)$. We have $\mu\left(\tilde{\Pi}_{T}\right) \leq \pi(p, q)+4 \varepsilon d / n$. 


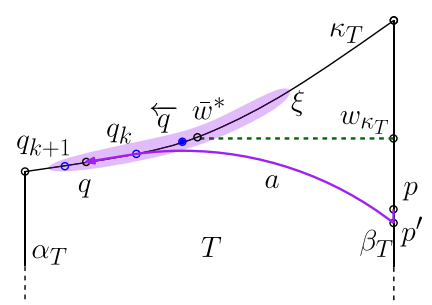

Fig. 13. Visualization of proof of Lemma 4.8.

Finally, suppose there is no locally reachable $q^{\prime}$ as described above. As in Section 3, let $\bar{w}^{*}$ denote the first intersection of well-behaved path $\gamma\left(p^{\prime}, q\right)$ with $\kappa_{T}$. Recall our algorithm adds sample points along several edgelets of length $O(d)$ such that each pair of samples lies at cost $\varepsilon d / n$ apart. By Lemma 4.6, point $q$ lies on one of these edgelets $\xi$.

By Lemma 3.3 and construction, either $\bar{w}^{*} \in \xi$ and $\bar{w}^{*}$ lies between consecutive sample points of $\xi$ we denoted as $\overleftarrow{q}$ and $\vec{q}$, or $\bar{w}^{*} \notin \xi$ and exactly one of $\overleftarrow{q}$ or $\vec{q}$ exists at an endpoint of $\xi$. By construction, each existing point of $\overleftarrow{q}$ and $\vec{q}$ is in $S\left(p^{\prime}\right)$. Let $q_{-} \in\{\overleftarrow{q}, \vec{q}\}$ be the first sample point of $\xi$ encountered as we walk along $\kappa_{T}$ from $\bar{w}^{*}$, past $q$, and to an endpoint of $\kappa_{T}$. We claim there

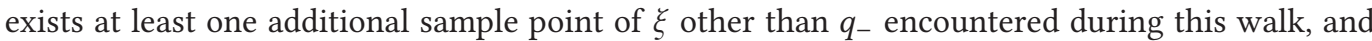
we denote $q_{0}$ as the first of these sample points. Indeed, if $q_{0}$ does not exist, then $\bar{w}^{*} \in \xi$ and $q$ lies between $\overleftarrow{q}$ and $\vec{q}$. At least one of them is locally reachable from $p^{\prime}$ by Lemma 4.7, which contradicts the assumption that $q$ is at least $\varepsilon d / n$ cost away from any sample point of $\xi \cap S\left(p^{\prime}\right)$ locally reachable from $p^{\prime}$. By a similar argument, we claim $q$ does not lie between $q_{0}$ and $\bar{w}^{*}$.

Recall, our algorithm adds samples $q_{i}$ to $S(p)$ spaced geometrically away from one of $\overleftarrow{q}$ and $\vec{q}$ in the direction of $q$; point $q_{0}$ is one of these samples. These samples also include one endpoint of $\xi$. Let $q_{k}, q_{k+1}$ be two consecutive sample points of $S(p)$ such that $q$ lies between them. By Lemma 4.7, at least one of $q_{k}$ and $q_{k+1}$ is locally reachable from $p^{\prime}$. Let $q^{\prime}$ be this locally reachable point. Let $a$ be the mimimal-cost path from $p^{\prime}$ to $q^{\prime}$. As before, we replace $\Pi^{*}[p, q]$ with $\tilde{\Pi}_{T}=p p^{\prime} \circ$ a $\circ \gamma\left(q^{\prime}, q\right)$. See Figure 13.

Let $\delta=\pi\left(q_{-}, q\right) n /(\varepsilon d)$. Value $\delta$ is an upper bound on the number of samples in $\xi$ between $q_{-}$ and $q$. We have $\left\lfloor(1+\varepsilon)^{k}\right\rfloor \leq \delta \leq\left\lfloor(1+\varepsilon)^{k+1}\right\rfloor$. In particular, $\delta \leq(1+\varepsilon)^{k+1}$, which implies $\delta-\lfloor(1+$ $\left.\varepsilon)^{k}\right\rfloor \leq \varepsilon \delta+1$. Similarly, $\left\lfloor(1+\varepsilon)^{k+1}\right\rfloor-\delta \leq \varepsilon \delta$. By Lemma 3.2, $\pi\left(q_{-}, q\right) \leq 11 \mu\left(p^{\prime}, q\right)$. We have

$$
\begin{aligned}
\pi\left(q, q^{\prime}\right) & \leq(\varepsilon \delta+1) \frac{\varepsilon d}{n} \\
& \leq\left(\pi\left(q_{-}, q\right) \frac{\varepsilon n}{\varepsilon d}+1\right) \frac{\varepsilon d}{n} \\
& =\varepsilon \pi\left(q_{-}, q\right)+\frac{\varepsilon d}{n} \\
& \leq 11 \varepsilon \pi\left(p^{\prime}, q\right)+\frac{\varepsilon d}{n} .
\end{aligned}
$$

We have $\pi\left(p^{\prime}, q^{\prime}\right) \leq \pi\left(p^{\prime}, q\right)+\pi\left(q, q^{\prime}\right) \leq(1+11 \varepsilon) \cdot \pi\left(p^{\prime}, q\right)+\varepsilon d / n$. Therefore, in all three cases we have

$$
\mu\left(\tilde{\Pi}_{T}\right) \leq(1+O(\varepsilon)) \pi(p, q)+O(\varepsilon d / n) .
$$

Summing over all steps in the deformation of $\Pi^{*}$ and using the fact $d^{*} \leq d \leq c d^{*}$ for a constant $c$, we obtain $\mu(\tilde{\Pi})=(1+O(\varepsilon)) d^{*}$. As before, it is clear from the construction that $\tilde{\Pi}$ is a path in $G_{3}$.

We conclude with our main theorem. 
Theorem 4.9. Let $\mathcal{O}$ be a set of disjoint polygonal obstacles in the plane with $n$ vertices total, and let $s, t$ be two points outside $\mathcal{O}$. Given a parameter $\varepsilon \in(0,1]$, there exists an $O\left(\frac{n^{2}}{\varepsilon^{2}} \log \frac{n}{\varepsilon}\right)$-time approximation algorithm for the minimum-cost path problem between $s$ and $t$ such that the algorithm returns an $s, t$-path of cost at most $(1+\varepsilon) \pi(s, t)$.

\section{DISCUSSION}

In this article, we present the first polynomial-time approximation algorithm for the problem of computing minimal-cost paths between two given points (when using the cost defined in Equation (1)). There are a few obvious directions in which our algorithm could be extended: (i) Can the running time of our algorithm be improved to near-linear? A possible approach would be to refine the notion of anchor points so it suffices to put only $O(\log n)$ additional points on each edge of the refined Voronoi diagram. (ii) Can the algorithm be extended to handle more general cost functions, where the cost of a path $\gamma$ is

$$
\mu(\gamma):=\int_{\gamma} \frac{1}{\operatorname{clr}(\gamma(\tau))^{c}} \mathrm{~d} \tau,
$$

and $c \geq 1$ is an integer? Although Lemma 3.2 extends to such cost functions, it is not clear whether Lemma 3.3 extends to this case. (iii) Does the algorithm work in an algebraic model of computation in which we assume that the roots of fixed-degree polyniomial can be computed in $O(1)$ time but we do not assume to compute a basic trigonometric function in $O(1)$ time? Since our goal is to compute a near-optimal path, it suffices to compute the cost of a path approximately. In principle, we can approximate a trigonometric function by a polynomial. Thus, a straightforward approach will be to introduce an additive approximation error. A more careful approach is needed if we wish to keep the relative approximation small without increasing the asymptotic running time of the algorithm.

Finally, there are other natural interesting open problems that we believe should be addressed. The first is to determine if the problem at hand is NP-hard. When considering the complexity of such a problem, one needs to consider both the algebraic complexity and the combinatorial complexity. In this case, we suspect that the algebraic complexity may be high because of the cost function we consider. However, we believe that combinatorial complexity, defined analogously to the number of "edge sequences," may be small. The second natural interesting open problem calls for extending our algorithm to compute near-optimal paths amid polyhedral obstacles in $\mathbb{R}^{3}$.

\section{ACKNOWLEDGMENTS}

We thank the referees for their useful comments on the article.

\section{REFERENCES}

[1] Pankaj K. Agarwal and Hongyan Wang. 2000. Approximation algorithms for curvature-constrained shortest paths. SIAM f. Comput. 30, 6 (2000), 1739-1772.

[2] Franz Aurenhammer, Rolf Klein, and Der-Tsai Lee. 2013. Voronoi Diagrams and Delaunay Triangulations. World Scientific.

[3] John F. Canny and John H. Reif. 1987. New lower bound techniques for robot motion planning problems. In Proceedings of the 28th Annual IEEE Symposium on Foundations of Computer Science. IEEE, 49-60.

[4] Nicholas J. Cavanna and Donald R. Sheehy. 2016. Adaptive metrics for adaptive samples. In Proceedings of the Canadian Conference on Computational Geometry. Simon Fraser University, Vancouver, British Columbia, Canada.

[5] Danny Z. Chen, Ovidiu Daescu, and Kevin S. Klenk. 2001. On geometric path query problems. Int. f. Comput. Geometry Appl. 11, 6 (2001), 617-645.

[6] Danny Z. Chen and Haitao Wang. 2013. Computing shortest paths among curved obstacles in the plane. In Proceedings of the 29th Annual IEEE Symposium on Foundations of Computer Science. IEEE, 369-378. 
[7] Howie Choset, Kevin M. Lynch, Seth Hutchinson, George Kantor, Wolfram Burgard, Lydia E. Kavraki, and Sebastian Thrun. 2005. Principles of Robot Motion: Theory, Algorithms, and Implementation. MIT Press.

[8] Kenneth L. Clarkson. 2006. Building triangulations using epsilon-nets. In ACM Symposium on Theory of Computing. 326-335.

[9] Michael B. Cohen, Brittany Terese Fasy, Gary L. Miller, Amir Nayyeri, Donald R. Sheehy, and Ameya Velingker. 2015. Approximating nearest neighbor distances. In Proceedings of the 14th Annual Symposium on Algorithms and Data Structures. Springer, 200-211.

[10] Michael L. Fredman and Robert Endre Tarjan. 1987. Fibonacci heaps and their uses in improved network optimization algorithms. F. ACM 34, 3 (1987), 596-615.

[11] Dan Halperin, Oren Salzman, and Micha Sharir. 2017. Algorithmic motion planning. In Handbook of Discrete and Computational Geometry (3rd ed.), C. D. Tóth, J. O’Rourke, and J. E. Goodman (Eds.). CRC Press LLC, 1311-1342.

[12] Monika Rauch Henzinger, Philip N. Klein, Satish Rao, and Sairam Subramanian. 1997. Faster shortest-path algorithms for planar graphs. 7. Comput. Syst. Sci. 55, 1 (1997), 3-23.

[13] John Hershberger, Subhash Suri, and Hakan Yıldız. 2013. A near-optimal algorithm for shortest paths among curved obstacles in the plane. In Proceedings of the 29th Annual Symposium on Computational Geometry. 359-368.

[14] Niel Lebeck, Thomas Mølhave, and Pankaj K. Agarwal. 2013. Computing highly occluded paths on a terrain. In Proceedings of the 21st Annual ACM SIGSPATIAL International Conference on Advances in Geographic Information Systems. ACM, 14-23.

[15] Joseph S. B. Mitchell. 2017. Shortest paths and networks. In Handbook of Discrete and Computational Geometry (3rd ed.), C. D. Tóth, J. O’Rourke, and J. E. Goodman (Eds.). CRC Press LLC, 811-848.

[16] Joseph S. B. Mitchell, Günter Rote, and Gerhard J. Woeginger. 1992. Minimum-link paths among obstacles in the plan. Algorithmica 8, 5 \& 6 (1992), 431-459.

[17] Colm Ó’Dúnlaing and Chee-Keng Yap. 1985. A "retraction" method for planning the motion of a disc. f. Algo. 6, 1 (1985), 104-111.

[18] Guang Song, Shawna Miller, and Nancy M. Amato. 2001. Customizing PRM roadmaps at query time. In Proceedings of the IEEE International Conference on Robotics and Automation. IEEE, 1500-1505.

[19] Ron Wein, Jur P. van den Berg, and Dan Halperin. 2007. The visibility-Voronoi complex and its applications. Comput. Geom. 36, 1 (2007), 66-87.

[20] Ron Wein, Jur P. van den Berg, and Dan Halperin. 2008. Planning high-quality paths and corridors amidst obstacles. I. f. Robot. Res. 27, 11-12 (2008), 1213-1231.

Received June 2017; revised May 2018; accepted May 2018 